Pacific

Journal of

Mathematics

ASYMPTOTIC BEHAVIOUR AT INFINITY OF THREE-DIMENSIONAL STEADY VISCOELASTIC FLOWS

Sergueï A. Nazarov, Adélia Sequeira, and Juha H. Videman 


\title{
ASYMPTOTIC BEHAVIOUR AT INFINITY OF THREE-DIMENSIONAL STEADY VISCOELASTIC FLOWS
}

\author{
Sergueï A. Nazarov, Adélia Sequeira, and Juha H. Videman
}

The steady motion of viscoelastic fluids is investigated in a three-dimensional exterior domain. Results concerning existence, uniqueness and asymptotic behaviour are obtained using appropriately constructed function spaces in which the elements are defined as a sum of the main asymptotic term and of the remainder living in a proper weighted Sobolev space. The equations are written as a coupled system that, at the first stage, can be studied as two linear problems composed of a Stokes system and a transport equation. Finally, a standard contraction argument provides existence and uniqueness of solutions for the original nonlinear coupled set of equations, when the data are sufficiently small.

\section{Introduction.}

The well-posedness of the equations governing the steady motion of incompressible viscoelastic fluids in two- and three-dimensional exterior domains has been investigated recently in several articles, cf. [7, 8, 22, 23, 32, 26]. These studies, as most of the other related works on viscoelastic fluids of differential and rate type, see e.g., $[\mathbf{2}, \mathbf{2 8}, \mathbf{9}, \mathbf{3}, \mathbf{2 5}]$ and all the references quoted therein, are crucially based on the mixed structure of the equations of motion. In fact, the equations form a system of nonlinear PDE's of elliptichyperbolic type which can most naturally be studied by suitably decoupling the elliptic and hyperbolic parts of the equations.

In this paper, we are concerned with the asymptotic behaviour of steady solutions for the equations of incompressible, homogeneous viscoelastic fluids in an exterior domain $\Omega \subset \mathbb{R}^{3}$. We assume that the flow domain $\Omega$ is an open and connected set, exterior to a compact set $\mathcal{B} \subset \mathbb{R}^{3}$, with a sufficiently smooth boundary $\partial \Omega$. Moreover, we suppose that the interior of $\mathcal{B}$ is nonempty and contains the origin of coordinates.

We shall focus on two particular models for viscoelastic fluids, namely:

Oldroyd-B fluid, where the extra-stress tensor $\mathbf{T}_{E}=p \mathbf{I}+\mathbf{T}$, with $\mathbf{T}$ denoting the Cauchy stress and $p$ the hydrostatic pressure, is related to the rate of 
deformation tensor $\mathbf{D}(\mathbf{v})=\frac{1}{2}\left(\nabla \mathbf{v}+(\nabla \mathbf{v})^{T}\right)$ by, cf. $[\mathbf{2 4}]$

$$
\begin{aligned}
& \mathbf{T}_{E}+\lambda_{1}\left(\frac{\partial}{\partial t} \mathbf{T}_{E}+\mathbf{v} \cdot \nabla \mathbf{T}_{E}-\nabla \mathbf{v} \mathbf{T}_{E}-\mathbf{T}_{E}(\nabla \mathbf{v})^{T}\right) \\
& =2 \mu_{0}\left(\mathbf{D}(\mathbf{v})+\lambda_{2}\left(\frac{\partial}{\partial t} \mathbf{D}(\mathbf{v})+\mathbf{v} \cdot \nabla \mathbf{D}(\mathbf{v})-\nabla \mathbf{v} \mathbf{D}(\mathbf{v})-\mathbf{D}(\mathbf{v})(\nabla \mathbf{v})^{T}\right)\right) .
\end{aligned}
$$

Second-grade fluid of Rivlin-Ericksen type, in which the extra-stress obeys the constitutive law, cf. $[4,31]$

$$
\begin{aligned}
\mathbf{T}_{E}=2 \mu \mathbf{D}(\mathbf{v})+2 \alpha_{1}\left(\frac{\partial}{\partial t} \mathbf{D}(\mathbf{v})\right. & +\mathbf{v} \cdot \nabla \mathbf{D}(\mathbf{v}) \\
& \left.+(\nabla \mathbf{v})^{T} \mathbf{D}(\mathbf{v})+\mathbf{D}(\mathbf{v}) \nabla \mathbf{v}\right)+4 \alpha_{2} \mathbf{D}^{2}(\mathbf{v}) .
\end{aligned}
$$

Here, $\mu, \lambda_{1}, \lambda_{2}, \alpha_{1}$ and $\alpha_{2}$ stand for material coefficients, $\mu_{0}, \mu>0$ are the fluid viscosities, $\lambda_{1}>0$ is the relaxation time, $\lambda_{2}$ the retardation time $\left(0<\lambda_{2}<\lambda_{1}\right)$, and $\alpha_{1}>0$ and $\alpha_{2}$ are called the normal stress moduli $\left(\alpha_{1}+\alpha_{2}=0\right)^{1}$.

Attaching the system of coordinates to $\mathcal{B}$, assuming that the fluid adheres to the fixed boundary of $\mathcal{B}$ and is at rest at infinity, and recalling the laws for the balance of linear momentum and for the conservation of mass, one obtains the following set of equations using the constitutive relation for the Oldroyd-B fluid

$$
\begin{cases}-\nu_{0}(1-\omega) \Delta \mathbf{v}+\nabla p=\nabla \cdot \boldsymbol{\tau}+\mathbf{v} \cdot \nabla \mathbf{v}+\mathbf{f} & \text { in } \Omega, \\ \nabla \cdot \mathbf{v}=0 & \text { on } \partial \Omega \\ \mathbf{v}=\mathbf{v}_{*}, \quad \mathbf{v}_{*} \cdot \mathbf{n}=0 & \\ \lim _{|x| \rightarrow \infty} \mathbf{v}(x)=0 & \text { in } \Omega . \\ \boldsymbol{\tau}+\lambda_{1} \mathbf{v} \cdot \nabla \boldsymbol{\tau}=2 \nu_{0} \omega \mathbf{D}(\mathbf{v})+\mathbf{N}_{1}(\boldsymbol{\tau}, \nabla \mathbf{v}) & \end{cases}
$$

Here, $\mathbf{v}=\left(v_{1}, v_{2}, v_{3}\right)$ is the velocity field, $p$ the associated pressure and $\boldsymbol{\tau}$ denotes the elastic part of the extra stress, i.e., $\mathbf{T}_{E}=2 \nu_{0}(1-\omega) \mathbf{D}(\mathbf{v})+\boldsymbol{\tau}$, where $\nu_{0}=\mu_{0} / \rho$ ( $\rho$ is the fluid density) and $\omega=1-\lambda_{2} / \lambda_{1}$. Furthermore, $\mathbf{f}$ is a prescribed vector field corresponding to the exterior body force, the nonlinear term $\mathbf{N}_{1}(\boldsymbol{\tau}, \nabla \mathbf{v})$ is given by

$$
\mathbf{N}_{1}(\boldsymbol{\tau}, \nabla \mathbf{v})=\lambda_{1}\left((\nabla \mathbf{v}) \boldsymbol{\tau}+\boldsymbol{\tau}(\nabla \mathbf{v})^{T}\right)
$$

and $\mathbf{n}$ denotes the unit outward normal vector.

\footnotetext{
${ }^{1}$ We tacitly assume the validity of all these thermodynamic conditions but point out that the mathematical results we prove here for second-grade fluids can be obtained without the restrictions $\alpha_{1}>0$ and $\alpha_{1}+\alpha_{2}=0$.
} 
For the second-grade fluid the equations of motion take the form

$(1.3)$

$$
\begin{cases}-\nu \Delta \mathbf{v}-\alpha \mathbf{v} \cdot \nabla \Delta \mathbf{v}+\nabla p=\mathbf{f}+\nabla \cdot\left(2 \alpha(\nabla \mathbf{v})^{T} \mathbf{D}(\mathbf{v})-\mathbf{v} \otimes \mathbf{v}\right) & \\ \nabla \cdot \mathbf{v}=0 & \text { in } \Omega \\ \mathbf{v}=\mathbf{v}_{*}, \quad \mathbf{v}_{*} \cdot \mathbf{n}=0 & \text { on } \partial \Omega \\ \lim _{|x| \rightarrow \infty} \mathbf{v}(x)=0 & \end{cases}
$$

where $\nu$ is the kinematic viscosity coefficient, $\alpha=\alpha_{1}$ is redefined after division by the constant density and $\otimes$ denotes the usual dyadic product.

The solvability of system (1.3) can be studied in the following decomposed form

$$
\begin{cases}-\Delta \mathbf{v}+\nabla \pi=\nabla \cdot \mathbf{z} & \text { in } \Omega, \\ \nabla \cdot \mathbf{v}=0 & \text { on } \partial \Omega, \\ \mathbf{v}=\mathbf{v}_{*}, \quad \mathbf{v}_{*} \cdot \mathbf{n}=0 & \\ \lim _{|x| \rightarrow \infty} \mathbf{v}(x)=0 & \\ \nu \mathbf{z}+\alpha \mathbf{v} \cdot \nabla \mathbf{z}=\mathbf{N}_{2}(\nabla \mathbf{v}, \pi, \mathbf{z})-\mathbf{v} \otimes \mathbf{v}+\mathcal{F} & \text { in } \Omega,\end{cases}
$$

with $\pi$ denoting a modified pressure, $\mathbf{z}$ standing for an auxiliary tensorvalued variable and $\mathcal{F}$ being a tensor field such that $\mathbf{f}=\nabla \cdot \mathcal{F}$. Moreover, the nonlinear term $\mathbf{N}_{2}(\nabla \mathbf{v}, \pi, \mathbf{z})$ is given by

$$
\mathbf{N}_{2}(\nabla \mathbf{v}, \pi, \mathbf{z})=\alpha\left(\mathbf{z}(\nabla \mathbf{v})^{T}+2(\nabla \mathbf{v})^{T} \mathbf{D}(\mathbf{v})-\pi(\nabla \mathbf{v})^{T}\right) .
$$

It is straightforward to show, see e.g., [23], that a sufficiently regular solution of (1.4) solves the original problem (1.3) with the pressure $p$ given by

$$
p=\nu \pi+\alpha \mathbf{v} \cdot \nabla \pi
$$

Moreover, it is a standard matter to prove that $\mathbf{f}$ can be written in a divergence-form without loss of generality (see also Lemma 5.2 for the same result in the functional setting of this paper).

The analysis of both systems (1.4) and (1.1) reduces to the study of the Stokes system

$$
\begin{cases}-\Delta \mathbf{v}+\nabla \pi=\boldsymbol{\psi} & \text { in } \Omega \\ \nabla \cdot \mathbf{v}=0 & \\ \mathbf{v}=\mathbf{v}_{*}, \quad \mathbf{v}_{*} \cdot \mathbf{n}=0 & \text { on } \partial \Omega\end{cases}
$$


for given vector fields $\boldsymbol{\psi}$ and $\mathbf{v}_{*}$, and of the transport equation

$$
\nu \mathbf{z}+\alpha \mathbf{v} \cdot \nabla \mathbf{z}=\mathbf{g} \text { in } \Omega,
$$

for a given tensor function $\mathbf{g}$ and a vector field $\mathbf{v}$, solenoidal in $\Omega$ and tangential on $\partial \Omega$.

Problem (1.3) has been recently studied in two different works, cf. [8, 23]. In $[8]$ the authors showed that provided the data is small enough there exists a solution to (1.3) such that

$$
\mathbf{v} \in L^{6}(\Omega)^{3}, \quad \nabla \mathbf{v} \in W^{2,2}(\Omega)^{3 \times 3}, \quad \nabla p \in L^{2}(\Omega)^{3}, \quad\left(p-p_{0}\right) \in L^{6}(\Omega),
$$

for some constant $p_{0}$. Here and in all that follows, we use the standard notations $L^{q}(\Omega), W^{m, q}(\Omega)$ and $C^{m, \delta}(\Omega)$, respectively for the Lebesgue, Sobolev and Hölder spaces.

In $[23]$ existence and uniqueness were proven, for small data, in a more restricted class of physically reasonable solutions. As for the Navier-Stokes equations, by physically reasonable one refers to sufficiently regular solutions having the decay, see $[\mathbf{5}, \mathbf{6}]$

$$
\mathbf{v}(x)=O\left(|x|^{-1}\right), \quad \nabla \mathbf{v}(x)=O\left(|x|^{-2}\right), \quad p(x)=O\left(|x|^{-2}\right) .
$$

The analysis of $[\mathbf{2 3}]$, based on the integral representation of the solution of the Stokes problem and on the $L^{2}(\Omega)$-estimates of the weakly singular integrals appearing in the representation formula, does not provide, however, optimal decay rates for the higher order derivatives of the solution $(\mathbf{v}, p)$. Moreover, with this method one cannot specify the asymptotic behaviour of the solution as $|x| \rightarrow \infty$. In particular, the method fails when applied to Equations (1.1) where the unknown functions are linearly related both through the Stokes and the transport equation.

Let us recall that the Navier-Stokes equations are not solvable in classical weighted Sobolev spaces while considering the convective term $\mathbf{v} \cdot \nabla \mathbf{v}$ as a perturbation of the Stokes problem. On the other hand, if one studies these equations in a function space in which the elements are defined as a sum of two parts, one of them containing the main asymptotics, then the NavierStokes problem is well-posed, see $[\mathbf{1 7}, \mathbf{1 8}]$. We also refer to $[\mathbf{1 5}, \mathbf{1 6}, \mathbf{2 0}]$ where these weighted spaces with detached asymptotics were applied to the study of the Navier-Stokes equations in other unbounded domains.

In this paper, we shall prove that problems (1.3) and (1.1) are well-posed in appropriately constructed weighted Sobolev spaces with detached asymptotics. In particular, we obtain accurate information about the asymptotic behaviour of the solutions. Let us point out that, although we consider here only two particular fluid models, our results remain valid for more general fluids of the Oldroyd and Rivlin-Ericksen type, for example for all the models studied in $[\mathbf{2 5}, \mathbf{3 2}]$. For the mechanical background of the rate and differential type viscoelastic fluids, we refer to $[31,29,27,10]$. 
Our paper is organized as follows. In Section 2, we study the Stokes operator in weighted spaces with detached asymptotics. In Section 3, we analyse the Newtonian and non-Newtonian nonlinear terms in these weighted Sobolev spaces. The transport equation is studied in Section 4 and, finally, in Section 5 we prove existence and uniqueness for the equations of viscoelastic fluids (1.1) and (1.3), using the results of the previous sections and a fixed point argument.

\section{Regarding the Stokes problem.}

We shall begin by studying the Stokes system in weighted Sobolev spaces. In order to apply the general theory for elliptic boundary-value problems in domains with conical points, see e.g., [19], we consider the problem with completely non-homogeneous right-hand side. After introducing the spaces, we recall the values of the weight, summability and regularity indices for which the Stokes operator is an isomorphism. Since the convective term $\mathbf{v} \cdot \nabla \mathbf{v}$ appearing in the nonlinear equations (1.1) and (1.3) is not continuous in the range space of the Stokes operator for those values of the indices, we construct particular function spaces in which the convective term can be treated. We end the section by re-analyzing the Stokes operator in these weighted Sobolev spaces with detached asymptotics.

2.1. The Stokes operator in weighted $L^{q}$-spaces. We shall use the following standard notation for the weighted Sobolev spaces. By $V_{\beta}^{l, q}(\Omega)$ we denote the closure of $C_{0}^{\infty}(\bar{\Omega})$ with respect to the weighted norm

$$
\left\|u ; V_{\beta}^{l, q}(\Omega)\right\|=\sum_{k=0}^{l}\left\|r^{\beta-l+k} D_{x}^{k} u ; L^{q}(\Omega)\right\|,
$$

where $l \in \mathbb{N}_{0}=\mathbb{N} \cup\{0\}, \beta \in \mathbb{R}, q \in(1, \infty)$ and $D_{x}^{k} u$ stands for the system of all $k$-th order derivatives of the function $u$. Let $W^{m, q}(\Omega)$, with $m \in \mathbb{Z}, q \in$ $(1, \infty)$, be the standard Sobolev space and $W^{l-1 / q, q}(\partial \Omega)$ denote the trace space on $\partial \Omega$ of functions both equipped with the usual norms. Since $\partial \Omega$ is compact and the origin of coordinates lays inside $\mathbb{R}^{3} \backslash \bar{\Omega}$, the trace space for $V_{\beta}^{l, q}(\Omega)$ coincides with $W^{l-1 / q, q}(\partial \Omega)$, for all $\beta \in \mathbb{R}$.

We associate to the Stokes problem

$$
\begin{cases}-\Delta \mathbf{v}+\nabla p=\mathbf{f}, \quad-\nabla \cdot \mathbf{v}=g & \text { in } \Omega, \\ \mathbf{v}=\mathbf{h} & \text { on } \partial \Omega,\end{cases}
$$

the mapping $\mathcal{S}_{\beta}^{l, q}: \mathcal{D}_{\beta}^{l, q} V(\Omega) \mapsto \mathcal{R}_{\beta}^{l, q} V(\Omega)$ defined by

$$
(\mathbf{v}, p) \mapsto(\mathbf{f}, g, \mathbf{h})=\mathcal{S}_{\beta}^{l, q}(\mathbf{v}, p),
$$


where

$$
\begin{aligned}
& \mathcal{D}_{\beta}^{l, q} V(\Omega) \equiv V_{\beta}^{l+1, q}(\Omega)^{3} \times V_{\beta}^{l, q}(\Omega), \\
& \mathcal{R}_{\beta}^{l, q} V(\Omega) \equiv V_{\beta}^{l-1, q}(\Omega)^{3} \times V_{\beta}^{l, q}(\Omega) \times W^{l+1-1 / q, q}(\partial \Omega)^{3} .
\end{aligned}
$$

The mapping $\mathcal{S}_{\beta}^{l, q}$ is continuous for any $q \in(1, \infty), l \in \mathbb{N}$ and $\beta \in \mathbb{R}$.

The following theorem can be derived from the general results proved in $[\mathbf{1 1}, \mathbf{1 3}, \mathbf{1 4}]$, see also [19] $§ 3.6,[30, \mathbf{1 7}, \mathbf{1 8}]$.

Theorem 2.1. The mapping (2.2) is an isomorphism if and only if

$$
\beta-l \in\left(1-\frac{3}{q}, 2-\frac{3}{q}\right) .
$$

Regarding the asymptotics of solutions, we recall the next theorem dating back to Kondratiev [11] and Maz'ya, Plamenevskii [14] (see also Theorems 4.2.1, 6.4.3 and Remark 4.1.5 in [19]).

Theorem 2.2. Let $(\mathbf{f}, g, \mathbf{h}) \in \mathcal{R}_{\gamma}^{l, q} V(\Omega) \subset \mathcal{R}_{\beta}^{l, q} V(\Omega)$ with

$$
\gamma-l \in\left(2-\frac{3}{q}, 3-\frac{3}{q}\right) \text {. }
$$

Then the solution $(\mathbf{v}, p) \in \mathcal{D}_{\beta}^{l, q} V(\Omega)$ can be expressed in the asymptotic form

$$
(\mathbf{v}, p)=\left(\mathbf{v}^{0}, p^{0}\right)+(\widetilde{\mathbf{v}}, \widetilde{p}),
$$

where $(\widetilde{\mathbf{v}}, \widetilde{p}) \in \mathcal{D}_{\gamma}^{l, q} V(\Omega)$ and

$$
\left(\mathbf{v}^{0}(x), p^{0}(x)\right)=b_{1} \mathbf{E}^{1}(x)+b_{2} \mathbf{E}^{2}(x)+b_{3} \mathbf{E}^{3}(x),
$$

with $\mathbf{E}^{j}$ denoting the $j$-th column of the fundamental matrix of the Stokes operator in $\mathbb{R}^{3}$ and $b_{j} \in \mathbb{R}, j=1,2,3$. Moreover, the following estimate holds

$$
\left\|(\widetilde{\mathbf{v}}, \widetilde{p}) ; \mathcal{D}_{\gamma}^{l, q} V(\Omega)\right\|+\left|b_{1}\right|+\left|b_{2}\right|+\left|b_{3}\right| \leq c\left\|(\mathbf{f}, g, \mathbf{h}) ; \mathcal{R}_{\gamma}^{l, q} V(\Omega)\right\| .
$$

2.2. The auxiliary problem on the unit sphere $\mathbb{S}^{2}$. In order to investigate the properties of the Stokes operator $\mathcal{S}_{\beta}^{l, q}$ in a more detailed way, let us consider the following Stokes system in the complete cone $\mathbb{R}^{3} \backslash\{0\}$

$$
-\Delta \mathbf{v}^{0}+\nabla p^{0}=\mathbf{f}^{0}, \quad-\nabla \cdot \mathbf{v}^{0}=g^{0}, \quad \text { in } \mathbb{R}^{3} \backslash\{0\}
$$

where

$$
\begin{array}{ll}
\mathbf{v}^{0}(x)=r^{\lambda} \mathbf{V}(\theta), & p^{0}(x)=r^{\lambda-1} P(\theta) \\
\mathbf{f}^{0}(x)=r^{\lambda-2} \mathbf{F}(\theta), & g^{0}(x)=r^{\lambda-1} G(\theta),
\end{array}
$$


$\mathbf{V}, P, \mathbf{F}$ and $G$ are functions on the sphere $\mathbb{S}^{2}, r=|x|, \theta=|x|^{-1} x \in \mathbb{S}^{2}$ and $\lambda \in \mathbb{C}$. Recall that $\Omega$ can be viewed as a domain with a conical point at infinity and that at large distances $\Omega$ coincides with the complete cone.

Substituting (2.7) into (2.6) and separating the radial variable, one obtains a system of differential equations written on the sphere in a compact form as, see e.g., [12]

$$
\mathcal{S}(\lambda)(\mathbf{V}, P)=(\mathbf{F}, G) \quad \text { on } \mathbb{S}^{2} .
$$

\section{Remark 2.1.}

(1) Note that the linear combination (2.5) is of the form $(2.7)_{1}$ with $\lambda=$ -1 . Moreover, $\left(\mathbf{v}^{0}, p^{0}\right)$ solves the homogeneous Stokes system (2.6).

(2) If $\lambda=0$ then any power solution of the type (2.7) 1 to the homogeneous Stokes system (2.6) has the form $\mathbf{V}=$ const, $P=0$.

Remark 2.2. The bounds for the difference $\beta-l$ in (2.3) can be obtained heuristically by requiring that any linear combination (2.5) belongs to the space $\mathcal{D}_{\beta}^{l, q} V(\Omega)$ while $(\mathbf{c}, 0) \notin \mathcal{D}_{\beta}^{l, q} V(\Omega)$ for any $\mathbf{c} \in \mathbb{R}^{3} \backslash\{0\}$.

Lemma 2.1 (see Lemma 3.5.9 [19]). The following Green's formula is valid for all smooth functions $\mathbf{V}, P$ and $\mathbf{W}, Q$ defined on the sphere $\mathbb{S}^{2}$

$$
(\mathcal{S}(\lambda)(\mathbf{V}, P),(\mathbf{W}, Q))_{\mathbb{S}^{2}}=((\mathbf{V}, P), \mathcal{S}(-1-\bar{\lambda})(\mathbf{W}, Q))_{\mathbb{S}^{2}},
$$

where $(\cdot, \cdot)_{\mathbb{S}^{2}}$ stands for the inner product in $L^{2}\left(\mathbb{S}^{2}\right)$ and $\bar{\lambda}$ is the complex conjugate of $\lambda$.

Let us consider system (2.8) with $\lambda=-1$. By virtue of the Green's formula, the operators $\mathcal{S}(-1)$ and $\mathcal{S}(0)$ are formally adjoint. Furthermore, by Remark 2.1(2)

$$
\operatorname{ker} \mathcal{S}(0)=\{(\mathbf{V}, P): P=0, \mathbf{V} \text { is constant }\} .
$$

Hence, defining the domain and range spaces for the Stokes operator on the unit sphere by

$$
\begin{aligned}
& \mathcal{D}^{s, q} W\left(\mathbb{S}^{2}\right)=W^{s+1, q}\left(\mathbb{S}^{2}\right)^{3} \times W^{s, q}\left(\mathbb{S}^{2}\right) \\
& \mathcal{R}^{s, q} W\left(\mathbb{S}^{2}\right)=W^{s-1, q}\left(\mathbb{S}^{2}\right)^{3} \times W^{s, q}\left(\mathbb{S}^{2}\right),
\end{aligned}
$$

one obtains the following assertion.

Lemma 2.2. Let $(\mathbf{F}, G) \in \mathcal{R}^{s, q} W\left(\mathbb{S}^{2}\right)$, with $s \in \mathbb{N}_{0}$. System (2.8), with $\lambda=-1$, has a solution $(\mathbf{V}, P) \in \mathcal{D}^{s, q} W\left(\mathbb{S}^{2}\right)$ if and only if the compatibility condition

$$
\int_{\mathbb{S}^{2}} \mathbf{F}(\theta) d s_{\theta}=0
$$

is satisfied. ${ }^{2}$

\footnotetext{
${ }^{2}$ Observe that (2.9) implies three scalar compatibility conditions.
} 
Remark 2.3. The solution $(\mathbf{V}(\theta), P(\theta))$ is defined up to an addendum $b_{1} \mathbf{E}^{1}(\theta)+b_{2} \mathbf{E}^{2}(\theta)+b_{3} \mathbf{E}^{3}(\theta)$ where $\mathbf{E}^{j}(\theta)$ denotes the trace of $\mathbf{E}^{j}(x)$ on the sphere $\mathbb{S}^{2}$. This follows from Theorem 2.2 and Remark 2.1.

Let us denote from now on by $\mathcal{R}^{s, q} W\left(\mathbb{S}^{2}\right)_{\perp}$ the subspace of $\mathcal{R}^{s, q} W\left(\mathbb{S}^{2}\right)$ consisting of functions that satisfy the compatibility condition (2.9).

2.3. The weighted spaces with detached asymptotics. Let $\mathfrak{D}_{\gamma}^{l, q} V(\Omega)$ denote a weighted Sobolev space of vector functions $(\mathbf{v}, p)$ admitting the representation

$$
(\mathbf{v}, p)=\left(r^{-1} \mathbf{V}(\theta), r^{-2} P(\theta)\right)+(\widetilde{\mathbf{v}}, \widetilde{p}),
$$

with

$$
(\mathbf{V}, P) \in \mathcal{D}^{l+1, q} W\left(\mathbb{S}^{2}\right), \quad(\widetilde{\mathbf{v}}, \widetilde{p}) \in \mathcal{D}_{\gamma}^{l, q} V(\Omega),
$$

and where the weight index $\gamma$ satisfies condition (2.4). The norm in $\mathfrak{D}_{\gamma}^{l, q} V(\Omega)$ is defined by

$$
\left\|(\mathbf{v}, p) ; \mathfrak{D}_{\gamma}^{l, q} V(\Omega)\right\|=\left\|(\widetilde{\mathbf{v}}, \widetilde{p}) ; \mathcal{D}_{\gamma}^{l, q} V(\Omega)\right\|+\left\|(\mathbf{V}, P) ; \mathcal{D}^{l+1, q} W\left(\mathbb{S}^{2}\right)\right\| .
$$

One easily proves the following result.

Lemma 2.3. For any $(\mathbf{v}, p) \in \mathfrak{D}_{\gamma}^{l, q} V(\Omega)$ it holds $(\mathbf{v}, p) \in \mathcal{D}_{\beta}^{l, q} V(\Omega)$, with $\beta$ satisfying (2.3). Furthermore, the estimate

$$
\left\|(\mathbf{v}, p) ; \mathcal{D}_{\beta}^{l, q} V(\Omega)\right\| \leq c\left\|(\mathbf{v}, p) ; \mathfrak{D}_{\gamma}^{l, q} V(\Omega)\right\|
$$

is valid.

Now, let us consider the Stokes problem (2.1) in the weighted space with detached asymptotics $\mathfrak{D}_{\gamma}^{l, q} V(\Omega)$. The range $\mathfrak{R}_{\gamma}^{l, q} V(\Omega)$ of the Stokes operator $\mathfrak{S}_{\gamma}^{l, q}$ acting on the domain $\mathfrak{D}_{\gamma}^{l, q} V(\Omega)$ is composed of triples $(\mathbf{f}, g, \mathbf{h})$ having the asymptotic form ${ }^{3}$

$$
\mathbf{f}(x)=r^{-3} \mathbf{F}(\theta)+\widetilde{\mathbf{f}}(x), \quad g(x)=r^{-2} G(\theta)+\widetilde{g}(x)
$$

and the finite norm

$$
\left\|(\mathbf{f}, g, \mathbf{h}) ; \mathfrak{R}_{\gamma}^{l, q} V(\Omega)\right\|=\left\|(\widetilde{\mathbf{f}}, \widetilde{g}, \mathbf{h}) ; \mathcal{R}_{\gamma}^{l, q} V(\Omega)\right\|+\left\|(\mathbf{F}, G) ; \mathcal{R}^{l+1, q} W\left(\mathbb{S}^{2}\right)\right\| .
$$

Theorem 2.3. Let $(\mathbf{f}, g, \mathbf{h}) \in \mathfrak{R}_{\gamma}^{l, q} V(\Omega)$. Problem (2.1) has a solution $(\mathbf{v}, p)$ $\in \mathfrak{D}_{\gamma}^{l, q} V(\Omega)$ if and only if the compatibility condition (2.9) is valid. This solution is unique and the estimate

$$
\left\|(\mathbf{v}, p) ; \mathfrak{D}_{\gamma}^{l, q} V(\Omega)\right\| \leq c\left\|(\mathbf{f}, g, \mathbf{h}) ; \mathfrak{R}_{\gamma}^{l, q} V(\Omega)\right\|
$$

holds.

\footnotetext{
${ }^{3}$ Observe that $\mathbf{h}$ has compact support.
} 
Proof. First, we look for a particular solution of the system (2.8) at $\lambda=-1$. Due to Lemma 2.2 and Remark 2.3 there exists a continuous inverse operator $\mathcal{R}$ for the mapping

$$
\mathcal{S}(-1): \mathcal{D}^{l+1, q} W\left(\mathbb{S}^{2}\right) \rightarrow \mathcal{R}^{l+1, q} W\left(\mathbb{S}^{2}\right)_{\perp} .
$$

We set $(\hat{\mathbf{V}}, \hat{P})=\mathcal{R}(\mathbf{F}, G)$ and conclude that

$$
\left\|(\hat{\mathbf{V}}, \hat{P}) ; \mathcal{D}^{l+1, q} W\left(\mathbb{S}^{2}\right)\right\| \leq c\left\|(\mathbf{F}, G) ; \mathcal{R}^{l+1, q} W\left(\mathbb{S}^{2}\right)\right\| .
$$

Next, we consider the Stokes problem (2.1) with the right-hand side

$$
(\mathbf{f}, g, \mathbf{h})=\left(\widetilde{\mathbf{f}}, \widetilde{g}, \mathbf{h}-r^{-1} \hat{\mathbf{V}}\right) .
$$

Note that since $\hat{\mathbf{V}} \in W^{l+2, q}\left(\mathbb{S}^{2}\right)^{3}$, we have $\left(\widetilde{\mathbf{f}}, \widetilde{g}, \mathbf{h}-r^{-1} \hat{\mathbf{V}}\right) \in \mathcal{R}_{\gamma}^{l, q} V(\Omega) \subset$ $\mathcal{R}_{\beta}^{l, q} V(\Omega)$ and

$$
\begin{aligned}
& \left\|\left(\widetilde{\mathbf{f}}, \widetilde{g}, \mathbf{h}-r^{-1} \hat{\mathbf{V}}\right) ; \mathcal{R}_{\gamma}^{l, q} V(\Omega)\right\| \\
& \leq\left\|(\widetilde{\mathbf{f}}, \widetilde{g}, \mathbf{h}) ; \mathcal{R}_{\gamma}^{l, q} V(\Omega)\right\|+c\left\|\hat{\mathbf{V}} ; W^{l+2, q}\left(\mathbb{S}^{2}\right)^{3}\right\| \\
& \leq c\left\|(\mathbf{f}, g, \mathbf{h}) ; \mathfrak{R}_{\gamma}^{l, q} V(\Omega)\right\| .
\end{aligned}
$$

Hence, from Theorems 2.1 and 2.2 it follows that Problem (2.1) with the right-hand side $\left(\widetilde{\mathbf{f}}, \widetilde{g}, \mathbf{h}-r^{-1} \hat{\mathbf{V}}\right)$ admits a unique solution $(\hat{\mathbf{v}}, \hat{p}) \in \mathcal{D}_{\beta}^{l, q} V(\Omega)$ which can be expressed in the form

$$
(\hat{\mathbf{v}}, \hat{p})=\sum_{j=1}^{3} \hat{b}_{j} \mathbf{E}^{j}(x)+(\widetilde{\mathbf{v}}, \widetilde{p})
$$

and satisfies the estimate

$$
\left\|(\widetilde{\mathbf{v}}, \widetilde{p}) ; \mathcal{D}_{\gamma}^{l, q} V(\Omega)\right\|+\sum_{i=1}^{3}\left|\hat{b}_{j}\right| \leq c\left\|\left(\widetilde{\mathbf{f}}, \widetilde{g}, \mathbf{h}-r^{-1} \hat{\mathbf{V}}\right) ; \mathcal{R}_{\gamma}^{l, q} V(\Omega)\right\| .
$$

Now, let us set

$$
(\mathbf{V}(\theta), P(\theta))=(\hat{\mathbf{V}}(\theta), \hat{P}(\theta))+\sum_{i=1}^{3} \hat{b}_{j} \mathbf{E}^{j}(\theta),
$$

and observe that the $\operatorname{sum}(\mathbf{v}, p)=\left(r^{-1} \hat{\mathbf{V}}, r^{-2} \hat{P}\right)+(\hat{\mathbf{v}}, \hat{p})$ is of the form $(2.10)$ and belongs to $\mathfrak{D}_{\gamma}^{l, q} V(\Omega)$. Estimate (2.12) follows directly from Formulae (2.15) and (2.17), and from estimates (2.13), (2.14), (2.16). The uniqueness is a consequence of the properties of the operator $\mathcal{S}_{\beta}^{l, q}$, see Theorem 2.1 and Lemma 2.3.

It remains to verify that the compatibility condition (2.9) is necessary. This follows from Lemma 3.5.11 in [19] which states that if (2.9) is not true 
then the solution $(\mathbf{v}, p) \in \mathcal{D}_{\beta}^{l, q} V(\Omega)$ takes the form

$$
(\mathbf{v}(x), p(x))=\log r \sum_{j=1}^{3} b_{j} \mathbf{E}^{j}(x)+\left(r^{-1} \mathbf{V}(\theta), r^{-2} P(\theta)\right)+(\widetilde{\mathbf{v}}(x), \widetilde{p}(x)),
$$

where $(\mathbf{V}, P) \in \mathcal{D}^{l, q} W\left(\mathbb{S}^{2}\right),(\widetilde{\mathbf{v}}, \widetilde{p}) \in \mathfrak{D}_{\gamma}^{l, q} V(\Omega)$ and $\mathbf{b}=\left(b_{1}, b_{2}, b_{3}\right)$ is a nontrivial vector in $\mathbb{R}^{3}$. Clearly, the solution given by (2.18) does not belong to $\mathfrak{D}_{\gamma}^{l, q} V(\Omega)$.

\section{Estimating the nonlinearities.}

The convective term $\mathbf{v} \cdot \nabla \mathbf{v}$ can be shown to be continuous in the weighted Sobolev space $V_{\gamma}^{l-1, q}(\Omega)$, when $\mathbf{v} \in V_{\gamma}^{l+1, q}$, provided $\gamma-l \geq 2-\frac{3}{q}$. However, as seen in the previous section for these values of $\gamma, l$ and $q$, the Stokes problem is not uniquely solvable. Here, we shall justify the choice of the weighted space with detached asymptotics $\mathfrak{D}_{\gamma}^{l, q} V(\Omega)$ by showing that $(\mathbf{v} \cdot \nabla \mathbf{v}, 0,0) \in \mathfrak{R}_{\gamma}^{l, q} V(\Omega)$ and that, moreover, the convective term satisfies the compatibility condition (2.9). We start by recalling embedding results in weighted Sobolev spaces.

Let $l \geq 0$ be an integer, $\alpha \in(0,1)$, and $\beta \in(-\infty, \infty)$. By $\Lambda_{\beta}^{l, \alpha}(\Omega)$ we denote the weighted Hölder space, i.e., the space of continuously differentiable functions up to the order $l$, for which the norm

$$
\left.\begin{array}{rl}
\left\|u ; \Lambda_{\beta}^{l, \alpha}(\Omega)\right\|= & \sum_{k=0}^{l} \sup _{x \in \Omega}|x|^{\beta-l-\alpha+k}\left|D_{x}^{k} u(x)\right| \\
& +\sup _{x \in \Omega}\left\{|x|^{\beta} \sup _{\substack{y \in \Omega \\
|x-y|<\frac{1}{2}|x|}}\left\{|x-y|^{-\alpha}\left|D_{x}^{l} u(x)-D_{y}^{l} u(y)\right|\right\}\right.
\end{array}\right\}
$$

is finite.

Lemma 3.1. Let $u \in V_{\beta}^{l, q}(\Omega)$.

(i) If $q l \leq 3$, with $q \leq t<\frac{3 q}{3-q l}$, then $u \in V_{\beta-l-\frac{3}{t}+\frac{3}{q}}^{0, t}(\Omega)$ and the estimate

$$
\left\|u ; V_{\beta-l-\frac{3}{t}+\frac{3}{q}}^{0, t}(\Omega)\right\| \leq c\left\|u ; V_{\beta}^{l, q}(\Omega)\right\|
$$

holds.

(ii) If $q l>3$ and $m+\alpha \leq l-\frac{3}{q}$ with $\alpha \in(0,1)$, then $u \in \Lambda_{m+\alpha+\beta-l+\frac{3}{q}}^{m, \alpha}(\Omega)$ and

$$
\left\|u ; \Lambda_{m+\alpha+\beta-l+\frac{3}{q}}^{m, \alpha}(\Omega)\right\| \leq c\left\|u ; V_{\beta}^{l, q}(\Omega)\right\| .
$$


Proof. See Maz'ya and Plamenevskii [14], Section 6.6 in Nazarov and Plamenevskii [19] and Borchers and Pileckas [1].

\subsection{The convective term.}

Lemma 3.2. Let $\left(\mathbf{v}^{i}, 0\right) \in \mathfrak{D}_{\gamma}^{l, q} V(\Omega)$, with $i=1,2, l \geq 1, \frac{3}{2}<q<\infty$ and $\gamma-l \in\left(2-\frac{3}{q}, 3-\frac{3}{q}\right)$. Then $\left(\mathbf{v}^{1} \cdot \nabla \mathbf{v}^{2}, 0,0\right) \in \mathfrak{R}_{\gamma}^{l, q} V(\Omega)$ and the following estimate holds

$$
\left\|\left(\mathbf{v}^{1} \cdot \nabla \mathbf{v}^{2}, 0,0\right) ; \mathfrak{R}_{\gamma}^{l, q} V(\Omega)\right\| \leq c\left\|\left(\mathbf{v}^{1}, 0\right) ; \mathfrak{D}_{\gamma}^{l, q} V(\Omega)\right\|\left\|\left(\mathbf{v}^{2}, 0\right) ; \mathfrak{D}_{\gamma}^{l, q} V(\Omega)\right\| .
$$

Proof. Let us note that we can clearly assume that $\Omega=\mathbb{R}^{3} \backslash \mathbb{B}_{1}$. In view of the representation formula (2.10) one has

$$
\begin{aligned}
\mathbf{v}^{1} \cdot \nabla \mathbf{v}^{2}= & \frac{1}{r} \mathbf{V}^{1}(\theta) \cdot \nabla_{x}\left(\frac{1}{r} \mathbf{V}^{2}(\theta)\right)+\widetilde{\mathbf{v}}^{1}(x) \cdot \nabla_{x}\left(\frac{1}{r} \mathbf{V}^{2}(\theta)\right) \\
& +\frac{1}{r} \mathbf{V}^{1}(\theta) \cdot \nabla_{x} \widetilde{\mathbf{v}}^{2}(x)+\widetilde{\mathbf{v}}^{1}(x) \cdot \nabla_{x} \widetilde{\mathbf{v}}^{2}(x) .
\end{aligned}
$$

Writing $\mathbf{V}^{1}(\theta)=\left(V_{r}^{1}(\theta), \mathbf{V}_{\theta}^{1}(\theta)\right)$, one easily sees that

$$
\frac{1}{r} \mathbf{V}^{1}(\theta) \cdot \nabla_{x}\left(\frac{1}{r} \mathbf{V}^{2}(\theta)\right)=\frac{1}{r^{3}}\left(-V_{r}^{1}(\theta)+\mathbf{V}_{\theta}^{1}(\theta) \nabla_{\theta}\right) \mathbf{V}^{2}(\theta)=\frac{1}{r^{3}} \mathbf{F}(\theta),
$$

and by Sobolev embeddings one obtains the estimate

$$
\left\|\mathbf{F} ; W^{l, q}\left(\mathbb{S}^{2}\right)^{3}\right\| \leq c\left\|\mathbf{V}^{1} ; W^{l+2, q}\left(\mathbb{S}^{2}\right)^{3}\right\|\left\|\mathbf{V}^{2} ; W^{l+2, q}\left(\mathbb{S}^{2}\right)^{3}\right\| .
$$

Now let us estimate the second term on the right-hand side of (3.2). We may write for all $k=0, \ldots, l-1$

$$
\begin{aligned}
& \left\|r^{\gamma-l+1+k} D_{x}^{k}\left(\widetilde{\mathbf{v}}^{1}(x) \cdot \nabla_{x}\left(\frac{1}{r} \mathbf{V}^{2}(\theta)\right)\right) ; L^{q}(\Omega)^{3}\right\|^{q} \\
& =\int_{1}^{\infty} r^{2} d r \int_{\mathbb{S}^{2}} r^{(\gamma-l+1+k) q}\left|D_{x}^{k}\left(r^{-2}\left(-\widetilde{v}_{r}^{1}(r, \theta)+\widetilde{\mathbf{v}}_{\theta}^{1}(r, \theta) \nabla_{\theta}\right) \mathbf{V}^{2}(\theta)\right)\right|^{q} d s_{\theta} \\
& \leq c\left\|\mathbf{V}^{2} ; C^{k+1}\left(\mathbb{S}^{2}\right)^{3}\right\|^{q} \sum_{j \leq k} \int_{1}^{\infty} r^{(\gamma-l-1+j) q} \int_{\mathbb{S}^{2}}\left|D_{x}^{j} \widetilde{\mathbf{v}}^{1}(r, \theta)\right|^{q} r^{2} d s_{\theta} d r \\
& \leq c\left\|\mathbf{V}^{2} ; C^{l, q}\left(\mathbb{S}^{2}\right)^{3}\right\|^{q}\left\|\widetilde{\mathbf{v}}^{1} ; V_{\gamma}^{l+1, q}(\Omega)^{3}\right\|^{q},
\end{aligned}
$$

where, for simplicity, $\widetilde{\mathbf{v}}^{1}$ denotes the solution written either in Cartesian or spherical coordinates and where we have set $\widetilde{\mathbf{v}}^{1}(r, \theta)=\left(\widetilde{v}_{r}^{1}(r, \theta), \widetilde{\mathbf{v}}_{\theta}^{1}(r, \theta)\right)$. From (3.4) it follows that

$$
\left\|\widetilde{\mathbf{v}}^{1}(x) \cdot \nabla_{x}\left(\frac{1}{r} \mathbf{V}^{2}(\theta)\right) ; V_{\gamma}^{l-1, q}(\Omega)^{3}\right\| \leq c\left\|\mathbf{V}^{2} ; W^{l+2, q}\left(\mathbb{S}^{2}\right)^{3}\right\|\left\|\widetilde{\mathbf{v}}^{1} ; V_{\gamma}^{l+1, q}(\Omega)^{3}\right\| .
$$


On the other hand, we readily get for all $k=0, \ldots, l-1$

$$
\begin{aligned}
& \left\|r^{\gamma-l+1+k} D_{x}^{k}\left(\frac{1}{r} \mathbf{V}^{1}(\theta) \cdot \nabla_{x} \widetilde{\mathbf{v}}^{2}(x)\right) ; L^{q}(\Omega)^{3}\right\|^{q} \\
& \leq c\left\|\mathbf{V}^{1} ; C^{k}\left(\mathbb{S}^{2}\right)^{3}\right\|^{q} \sum_{j \leq k} \int_{1}^{\infty} r^{(\gamma-l+j) q}\left|D_{x}^{j}\left(\nabla_{x} \widetilde{\mathbf{v}}^{2}(x)\right)\right|^{q} r^{2} d s_{\theta} d r \\
& \leq c\left\|\mathbf{V}^{1} ; W^{l+1, q}\left(\mathbb{S}^{2}\right)^{3}\right\|^{q} \sum_{j \leq k+1} \int_{1}^{\infty} r^{(\gamma-l+j) q} \int_{\mathbb{S}^{2}}\left|D_{x}^{j} \widetilde{\mathbf{v}}^{2}(x)\right|^{q} r^{2} d s_{\theta} d r \\
& \leq c \| \mathbf{V}^{1} ; W^{l+1, q}\left(\mathbb{S}^{3}\left\|^{q}\right\| \widetilde{\mathbf{v}}^{2} ; V_{\gamma}^{l, q}(\Omega)^{3} \|^{q} .\right.
\end{aligned}
$$

Finally, let us note that $\widetilde{\mathbf{v}} \in V_{\gamma}^{l+1, q}(\Omega)^{3}$ if and only if $r^{\gamma-l-1+3 / q} \widetilde{\mathbf{v}}(r, \theta) \in$ $W^{l+1, q}\left(\mathbb{Q}_{1}\right)^{3}$, where $\mathbb{Q}_{1}=(1, \infty) \times \mathbb{S}^{2}$. In fact, see also Section 3.5.1 in [19], we can write for all $k=0, \ldots, l+1$

$$
\begin{aligned}
& \int_{1}^{\infty} r^{(\gamma-l-1+k) q} \int_{\mathbb{S}^{2}}\left|D_{x}^{k} \widetilde{\mathbf{v}}\right|^{q} r^{2} d r d s_{\theta} \\
& \simeq \sum_{j+p \leq k} \int_{1}^{\infty} \int_{\mathbb{S}^{2}} r^{(\gamma-l-1) q+3}\left|\left(r D_{r}\right)^{j} D_{\theta}^{p} \widetilde{\mathbf{v}}\right|^{q} \frac{d r}{r} d s_{\theta} \\
& \simeq \sum_{j+p \leq k} \int_{1}^{\infty} \int_{\mathbb{S}^{2}}\left|\left(r D_{r}\right)^{j} D_{\theta}^{p}\left(r^{\gamma-l-1+3 / q} \widetilde{\mathbf{v}}\right)\right|^{q} \frac{d r}{r} d s_{\theta},
\end{aligned}
$$

where the symbol $a \simeq b$ means that $c b \leq a \leq C b$ with some positive constants $c$ and $C$. Hence, using the Euler change of variables $t=\log r$, we conclude that

$$
\begin{aligned}
& \int_{1}^{\infty} r^{(\gamma-l-1+k) q} \int_{\mathbb{S}^{2}}\left|D_{x}^{k} \widetilde{\mathbf{v}}(r, \theta)\right|^{q} r^{2} d r d s_{\theta} \\
& \simeq \sum_{j+p \leq k} \int_{1}^{\infty} \int_{\mathbb{S}^{2}}\left|D_{t}^{j} D_{\theta}^{p}\left(e^{(\gamma-l-1+3 / q) t} \widetilde{\mathbf{v}}\left(e^{t}, \theta\right)\right)\right|^{q} d t d s_{\theta} \\
& \simeq \sum_{j+p \leq k} \int_{1}^{\infty} \int_{\mathbb{S}^{2}}\left|e^{(\gamma-l-1+3 / q) t} D_{t}^{j} D_{\theta}^{p} \widetilde{\mathbf{v}}\left(e^{t}, \theta\right)\right|^{q} d t d s_{\theta},
\end{aligned}
$$

which implies that $\left\|\widetilde{\mathbf{v}} ; V_{\gamma}^{l+1, q}(\Omega)^{3}\right\| \simeq\left\|r^{\gamma-l-1+3 / q} \widetilde{\mathbf{v}}(r, \theta) ; W^{l+1, q}\left(\mathbb{Q}_{1}\right)^{3}\right\|$. Now, let

$$
\hat{\mathbf{v}}(t, \theta)=\left.r^{\gamma-l-1+3 / q} \widetilde{\mathbf{v}}(r, \theta)\right|_{r=e^{t}}
$$

and consider the term $\widetilde{\mathbf{v}}^{1} \cdot \nabla_{x} \widetilde{\mathbf{v}}^{2}$. One easily sees that

$$
\begin{aligned}
& r^{\gamma-l+1+3 / q} \widetilde{\mathbf{v}}^{1} \cdot \nabla_{x} \widetilde{\mathbf{v}}^{2} \\
& \simeq r^{2-(\gamma-l-1+3 / q)} r^{\gamma-l-1+3 / q} \widetilde{\mathbf{v}}^{1} \cdot\left(\partial_{r}, r^{-1} \nabla_{\theta}\right)\left(r^{\gamma-l-1+3 / q} \widetilde{\mathbf{v}}^{2}\right) \\
& =e^{-(\gamma-l-2+3 / q) t} \hat{\mathbf{v}}^{1}(t, \theta) \cdot\left(\partial_{t}, \nabla_{\theta}\right) \hat{\mathbf{v}}^{2}(t, \theta) .
\end{aligned}
$$


Now, since $\hat{\mathbf{v}}^{i} \in W^{l+1, q}\left(\mathbb{Q}_{1}\right)^{3}, i=1,2, q>\frac{3}{2}$ and $\gamma-l>2-3 / q$, one concludes using embedding results that

$$
e^{-(\gamma-l-2+3 / q) t} \hat{\mathbf{v}}^{1}(t, \theta) \cdot \nabla_{x} \hat{\mathbf{v}}^{2}(t, \theta) \in W^{l-1, q}\left(\mathbb{Q}_{1}\right)^{3} .
$$

Consequently $\widetilde{\mathbf{v}}^{1} \cdot \nabla_{x} \widetilde{\mathbf{v}}^{2} \in V_{\gamma}^{l-1, q}(\Omega)^{3}$ and

$$
\left\|\widetilde{\mathbf{v}}^{1} \cdot \nabla_{x} \widetilde{\mathbf{v}}^{2} ; V_{\gamma}^{l-1, q}(\Omega)^{3}\right\| \leq c\left\|\widetilde{\mathbf{v}}^{1} ; V_{\gamma}^{l+1, q}(\Omega)^{3}\right\|\left\|\widetilde{\mathbf{v}}^{2} ; V_{\gamma}^{l+1, q}(\Omega)^{3}\right\| .
$$

From (3.3), (3.5), (3.6) and (3.8) one concludes that $\left(\mathbf{v}^{1} \cdot \nabla \mathbf{v}^{2}, 0,0\right) \in$ $\mathfrak{R}_{\gamma}^{l, q} V(\Omega)$ and that estimate (3.1) holds.

Next, let $\mathfrak{T}_{\gamma}^{l, q}(\Omega)$ denote the space of tensor functions $\mathbf{z}$ admitting the representation

$$
\mathbf{z}(x)=r^{-2} \mathbf{Z}(\theta)+\widetilde{\mathbf{z}}(x),
$$

with

$$
\left\|\mathbf{z} ; \mathfrak{T}_{\gamma}^{l, q}(\Omega)\right\|=\left\|\widetilde{\mathbf{z}} ; V_{\gamma}^{l, q}(\Omega)^{3 \times 3}\right\|+\left\|\mathbf{Z} ; W^{l+1, q}\left(\mathbb{S}^{2}\right)^{3 \times 3}\right\| .
$$

Lemma 3.3. Let $(\mathbf{v}, 0) \in \mathfrak{D}_{\gamma}^{l, q} V(\Omega)$, with $l \geq 1, \frac{3}{2}<q<\infty$ and $\gamma-l \in$ $\left(2-\frac{3}{q}, 3-\frac{3}{q}\right)$. Then $\mathbf{v} \otimes \mathbf{v} \in \mathfrak{T}_{\gamma}^{l, q}(\Omega)$ and the following estimates holds

$$
\left\|\mathbf{v} \otimes \mathbf{v} ; \mathfrak{T}_{\gamma}^{l, q}(\Omega)\right\| \leq c\left\|(\mathbf{v}, 0) ; \mathfrak{D}_{\gamma}^{l, q} V(\Omega)\right\|^{2} .
$$

Proof. First, let us write

$$
\begin{aligned}
\mathbf{v}(x) \otimes \mathbf{v}(x)=r^{-2} \mathbf{V}(\theta) & \otimes \mathbf{V}(\theta) \\
& +r^{-1}(\mathbf{V}(\theta) \otimes \widetilde{\mathbf{v}}(x)+\widetilde{\mathbf{v}}(x) \otimes \mathbf{V}(\theta))+\widetilde{\mathbf{v}}(x) \otimes \widetilde{\mathbf{v}}(x) .
\end{aligned}
$$

Now, since $q>\frac{3}{2}$, one readily gets by Sobolev embeddings

$$
\begin{aligned}
& \left\|\mathbf{V}(\theta) \otimes \mathbf{V}(\theta) ; W^{l+1, q}\left(\mathbb{S}^{2}\right)^{3 \times 3}\right\| \leq c\left\|\mathbf{V}(\theta) ; W^{l+2, q}\left(\mathbb{S}^{2}\right)^{3}\right\|^{2}, \\
& \left\|r^{-1}(\mathbf{V}(\theta) \otimes \widetilde{\mathbf{v}}(x)+\widetilde{\mathbf{v}}(x) \otimes \mathbf{V}(\theta)) ; V_{\gamma}^{l, q}(\Omega)^{3 \times 3}\right\| \\
& \leq c\left\|\mathbf{V}(\theta) ; W^{l+2, q}\left(\mathbb{S}^{2}\right)^{3}\right\|\left\|\widetilde{\mathbf{v}} ; V_{\gamma}^{l+1, q}(\Omega)^{3}\right\| .
\end{aligned}
$$

In order to show that $\widetilde{\mathbf{v}}(x) \otimes \widetilde{\mathbf{v}}(x) \in V_{\gamma}^{l, q}(\Omega)^{3 \times 3}$, we observe that

$$
r^{\gamma-l+3 / q} \widetilde{\mathbf{v}}(r, \theta) \otimes \widetilde{\mathbf{v}}(r, \theta) \simeq r^{-(\gamma-l-2+3 / q)} r^{\gamma-l-1+3 / q} \widetilde{\mathbf{v}} \otimes\left(r^{\gamma-l-1+3 / q} \widetilde{\mathbf{v}}\right) .
$$

Hence, defining $\hat{\mathbf{v}}(t, \theta)$ as in (3.7), taking into account that $\gamma-l>2-3 / q$ and $q>\frac{3}{2}$ and using Sobolev embeddings, one easily sees that

$$
r^{\gamma-l+3 / q} \widetilde{\mathbf{v}}(r, \theta) \otimes \widetilde{\mathbf{v}}(r, \theta) \simeq e^{-(\gamma-l-2+3 / q) t} \hat{\mathbf{v}}(t, \theta) \otimes \hat{\mathbf{v}}(t, \theta) \in W^{l, q}\left(\mathbb{Q}_{1}\right)^{3 \times 3} .
$$

This implies that $\widetilde{\mathbf{v}}(x) \otimes \widetilde{\mathbf{v}}(x) \in V_{\gamma}^{l, q}(\Omega)^{3 \times 3}$. 
Lemma 3.4. Assume that the vector function $\mathbf{F}(\theta) \in W^{l-1, q}(\mathbb{S})^{3}$ satisfies

$$
r^{-3} \mathbf{F}(\theta)=\nabla \cdot\left(r^{-2} \mathbf{G}(\theta)\right)
$$

with some $\mathbf{G} \in W^{l, q}(\mathbb{S})^{3 \times 3}$. Then $\int_{\mathbb{S}^{2}} \mathbf{F}(\theta) d s_{\theta}=0$.

Proof. Consider, for arbitrary $R>0$, the integral

$$
I=\int_{R}^{2 R} \int_{\mathbb{S}^{2}} r^{-3} \mathbf{F}(\theta) r^{2} d r d s_{\theta}=\ln 2 \int_{\mathbb{S}^{2}} \mathbf{F}(\theta) d s_{\theta} .
$$

In view of (3.9), one obtains

$$
\begin{aligned}
I & =\int_{\mathbb{B}_{2 R} \backslash \mathbb{B}_{R}} \nabla \cdot\left(r^{-2} \mathbf{G}(\theta)\right) d x \\
& =\int_{\partial\left(\mathbb{B}_{2 R} \backslash \mathbb{B}_{R}\right)} r^{-2} \mathbf{G}(\theta) \cdot \mathbf{n} d s \\
& =\int_{\mathbb{S}_{2 R}^{2}} r^{-2} \mathbf{G}(\theta) \cdot \frac{x}{|x|} d s_{x}-\int_{\mathbb{S}_{R}^{2}} r^{-2} \mathbf{G}(\theta) \cdot \frac{x}{|x|} d s_{x}
\end{aligned}
$$

where $\mathbf{n}$ denotes the unit outward normal vector. Since the integrands in the last two integrals in (3.10) are homogeneous functions of order -2 in $r$, these two integrals are equal to each other and one concludes that $I=0$.

Corollary 3.1. Let $(\mathbf{v}, 0) \in \mathfrak{D}_{\gamma}^{l, q} V(\Omega)$, with $l \geq 1, \frac{3}{2}<q<\infty$ and $\gamma-l \in$ $\left(2-\frac{3}{q}, 3-\frac{3}{q}\right)$. Then the convective term $\mathbf{v} \cdot \nabla \mathbf{v}$ satisfies the compatibility condition (2.9).

Proof. By Lemma 3.2, it holds $(\mathbf{v} \cdot \nabla \mathbf{v}, 0,0) \in \mathfrak{R}_{\gamma}^{l, q} V(\Omega)$ with the main asymptotic term given by

$$
\frac{1}{r^{3}}\left(-V_{r}(\theta)+V_{\theta}(\theta) \nabla_{\theta}\right) \mathbf{V}(\theta)=\frac{1}{r} \mathbf{V}(\theta) \cdot \nabla\left(\frac{1}{r} \mathbf{V}(\theta)\right) .
$$

On the other hand, since $\nabla \cdot \mathbf{v}=0$ implies that $\nabla \cdot\left(r^{-1} \mathbf{V}(\theta)\right)=0$, one can write

$$
\frac{1}{r} \mathbf{V}(\theta) \cdot \nabla\left(\frac{1}{r} \mathbf{V}(\theta)\right)=\nabla \cdot\left(\frac{1}{r^{2}} \mathbf{V}(\theta) \otimes \mathbf{V}(\theta)\right),
$$

and the previous lemma applies.

3.2. Non-Newtonian nonlinearities. In order to estimate the nonlinear terms $\mathbf{N}_{1}(\boldsymbol{\tau}, \nabla \mathbf{v})$ and $\mathbf{N}_{2}(\nabla \mathbf{v}, \pi, \mathbf{z})$ defined in (1.2) and (1.5), we need the following lemma.

Lemma 3.5. Let $\mathbf{g}, \mathbf{h} \in \mathfrak{T}_{\gamma}^{l, q}(\Omega)$, with $l \geq 2, q>\frac{3}{2}$ and assume that $\gamma-l \in$ $\left(2-\frac{3}{q}, 3-\frac{3}{q}\right)$. Then, $\mathbf{g h} \in V_{\gamma}^{l, q}(\Omega)^{3 \times 3}$ and the estimate

$$
\left\|\mathbf{g h} ; V_{\gamma}^{l, q}(\Omega)^{3 \times 3}\right\| \leq c\left\|\mathbf{g} ; \mathfrak{T}_{\gamma}^{l, q}(\Omega)\right\|\left\|\mathbf{h} ; \mathfrak{T}_{\gamma}^{l, q}(\Omega)\right\|
$$

holds. 
Proof. Since $\mathbf{g}, \mathbf{h} \in \mathfrak{T}_{\gamma}^{l, q}(\Omega)$, we can write

$$
\mathbf{g}(x)=r^{-2} \mathbf{G}(\theta)+\widetilde{\mathbf{g}}(x), \quad \mathbf{h}(x)=r^{-2} \mathbf{H}(\theta)+\widetilde{\mathbf{h}}(x),
$$

where $\mathbf{G}, \mathbf{H} \in W^{l+1, q}\left(\mathbb{S}^{2}\right)^{3 \times 3}$ and $\widetilde{\mathbf{g}}, \widetilde{\mathbf{h}} \in V_{\gamma}^{l, q}(\Omega)^{3 \times 3}$. Now, using the fact that $\gamma-l<3-\frac{3}{q}$, one readily obtains

$$
r^{-2} \mathbf{G}(\theta) \in V_{\gamma}^{l+1, q}(\Omega)^{3 \times 3} \hookrightarrow \Lambda_{\gamma}^{l+1-\frac{3}{q}-\alpha, \alpha}(\Omega)^{3 \times 3},
$$

with $\alpha \in(0,1)$, and where the last embedding follows from Lemma 3.1 (ii). In particular, there exists $\alpha \in(0,1)$ such that $r^{-2} \mathbf{G}(\theta) \in C_{0}^{l-1, \alpha}(\Omega)^{3 \times 3}$. In the same manner, one easily verifies the inclusions

$$
V_{\gamma}^{l, q}(\Omega) \hookrightarrow \Lambda_{\gamma}^{l-\frac{3}{q}-\alpha, \alpha}(\Omega) \hookrightarrow C_{0}^{l-2, \alpha}(\Omega),
$$

for some $\alpha \in(0,1)$. Moreover, it holds

$$
\begin{aligned}
\left\|r^{-2} \mathbf{G}(\theta) ; C_{0}^{l-1, \alpha}(\Omega)^{3 \times 3}\right\| & \leq c\left\|r^{-2} \mathbf{G}(\theta) ; V_{\gamma}^{l+1, q}(\Omega)^{3 \times 3}\right\| \\
& \leq c\left\|\mathbf{G}(\theta) ; W^{l+1, q}\left(\mathbb{S}^{2}\right)^{3 \times 3}\right\|, \\
\left\|\widetilde{\mathbf{g}} ; C_{0}^{l-2, \alpha}(\Omega)^{3 \times 3}\right\| & \leq c\left\|\widetilde{\mathbf{g}} ; V_{\gamma}^{l, q}(\Omega)^{3 \times 3}\right\| .
\end{aligned}
$$

Since $l \geq 2$, it follows that the terms $r^{-2} \mathbf{G}(\theta) \widetilde{\mathbf{h}}(x), r^{-2} \mathbf{H}(\theta) \widetilde{\mathbf{g}}(x)$, and $r^{-2} \mathbf{G}(\theta) r^{-2} \mathbf{H}(\theta)$ belong to $V_{\gamma}^{l, q}(\Omega)^{3 \times 3}$. Finally, since $q>\frac{3}{2}$ one obtains from Lemma 3.1 (i) the embedding $V_{\gamma}^{1, q}(\Omega) \hookrightarrow V_{\gamma-1+\frac{3}{2 q}}^{0,2 q}(\Omega)$, which together with (3.11) shows that $\widetilde{\mathbf{g}} \widetilde{\mathbf{h}} \in V_{\gamma}^{l, q}(\Omega)^{3 \times 3}$, for all $l \geq 2$.

Now, observe that if $(\mathbf{v}, p) \in \mathfrak{D}_{\gamma}^{l, q} V(\Omega)$ then in particular $\nabla \mathbf{v} \in \mathfrak{T}_{\gamma}^{l, q}(\Omega)$ and $p$ is a scalar function having the same asymptotic structure, and regularity and decay properties as functions in $\mathfrak{T}_{\gamma}^{l, q}(\Omega)$. Hence, by the previous lemma one arrives at the following two corollaries.

Corollary 3.2. Let $l \geq 2, q>\frac{3}{2}$ and $\gamma-l \in\left(2-\frac{3}{q}, 3-\frac{3}{q}\right)$ and assume that $(\mathbf{v}, 0) \in \mathfrak{D}_{\gamma}^{l, q} V(\Omega)$ and $\boldsymbol{\tau} \in \mathfrak{T}_{\gamma}^{l, q}(\Omega)$. Then $\mathbf{N}_{1}(\boldsymbol{\tau}, \nabla \mathbf{v}) \in V_{\gamma}^{l, q}(\Omega)^{3 \times 3}$ and

$$
\left\|\mathbf{N}_{1}(\boldsymbol{\tau}, \nabla \mathbf{v}) ; V_{\gamma}^{l, q}(\Omega)^{3 \times 3}\right\| \leq c\left\|(\mathbf{v}, 0) ; \mathfrak{D}_{\gamma}^{l, q} V(\Omega)\right\|\left\|\boldsymbol{\tau} ; \mathfrak{T}_{\gamma}^{l, q}(\Omega)\right\| .
$$

Corollary 3.3. Let $l \geq 2, q>\frac{3}{2}$ and $\gamma-l \in\left(2-\frac{3}{q}, 3-\frac{3}{q}\right)$ and assume that $(\mathbf{v}, \pi) \in \mathfrak{D}_{\gamma}^{l, q} V(\Omega)$ and $\mathbf{z} \in \mathfrak{T}_{\gamma}^{l, q}(\Omega)$. Then $\mathbf{N}_{2}(\nabla \mathbf{v}, \pi, \mathbf{z}) \in V_{\gamma}^{l, q}(\Omega)^{3 \times 3}$ and

$$
\begin{aligned}
& \left\|\mathbf{N}_{2}(\nabla \mathbf{v}, \pi, \mathbf{z}) ; V_{\gamma}^{l, q}(\Omega)^{3 \times 3}\right\| \\
& \leq c\left(\left\|(\mathbf{v}, \pi) ; \mathfrak{D}_{\gamma}^{l, q} V(\Omega)\right\|^{2}+\left\|(\mathbf{v}, \pi) ; \mathfrak{D}_{\gamma}^{l, q} V(\Omega)\right\|\left\|\mathbf{z} ; \mathfrak{T}_{\gamma}^{l, q}(\Omega)\right\|\right) .
\end{aligned}
$$




\section{Regarding the transport equation.}

Let us start by considering a model transport equation

$$
\mathbf{z}+\lambda_{1} \mathbf{v} \cdot \nabla \mathbf{z}=\mathbf{g} \quad \text { in } \Omega
$$

in usual Sobolev spaces, where $\mathbf{v}$ is a solenoidal vector field satisfying $\mathbf{v}=\mathbf{v}_{*}$, with $\mathbf{v}_{*} \cdot \mathbf{n}=0$ on $\partial \Omega$. The following lemma was proved in [21].

Lemma 4.1. Let $\mathbf{g} \in W^{l, q}(\Omega)^{3 \times 3}$, with $l \geq 2$ and $\frac{3}{2}<q<\infty$. Moreover, assume that $\mathbf{v} \in C^{l-1}(\bar{\Omega})^{3}$ and $D_{x}^{l} \mathbf{v} \in L^{3}(\Omega)^{3}$.

There exists $\varepsilon>0$ such that provided

$$
\left\|\mathbf{v} ; C^{l-1}(\bar{\Omega})^{3}\right\|+\left\|D_{x}^{l} \mathbf{v} ; L^{3}(\Omega)^{3}\right\| \leq \varepsilon,
$$

problem (4.1) admits a unique solution $\mathbf{z} \in W^{l, q}(\Omega)^{3 \times 3}$ and the estimate

$$
\left\|\mathbf{z} ; W^{l, q}(\Omega)^{3 \times 3}\right\| \leq c\left\|\mathbf{g} ; W^{l, q}(\Omega)^{3 \times 3}\right\|
$$

is valid.

Next, let us consider the transport equation (4.1) in the space $\mathfrak{T}_{\gamma}^{l, q}(\Omega)$. Let us assume that $\mathbf{g} \in \mathfrak{T}_{\gamma}^{l, q}(\Omega)$ and write

$$
\mathbf{g}(x)=r^{-2} \mathbf{G}(\theta)+\widetilde{\mathbf{g}}(x) .
$$

One can clearly predict that $\mathbf{z}$ has the asymptotic form

$$
\mathbf{z}(x)=r^{-2} \mathbf{G}(\theta)+\widetilde{\mathbf{z}}(x) .
$$

Now, let us prove that the remainder $\widetilde{\mathbf{z}}$, satisfying the transport equation

$$
\widetilde{\mathbf{z}}+\lambda_{1} \mathbf{v} \cdot \nabla \widetilde{\mathbf{z}}=\widetilde{\mathbf{g}}-\lambda_{1} \mathbf{v} \cdot \nabla\left(r^{-2} \mathbf{G}(\theta)\right) \quad \text { in } \Omega,
$$

belongs to the space $V_{\gamma}^{l, q}(\Omega)^{3 \times 3}$.

Remark 4.1. In order to be able to handle the term $\lambda_{1} \mathbf{v} \cdot \nabla\left(r^{-2} \mathbf{G}(\theta)\right)$ on the right hand side of (4.2), we need to assume here that the main asymptotic part defined on the sphere is more regular than the remainder. This assumption, which is only possible because $\mathbf{Z}(\theta)=\mathbf{G}(\theta)$, affects the definitions of all the function spaces with detached asymptotics, see e.g., (2.10), (2.11).

Lemma 4.2. Let $\mathbf{g} \in \mathfrak{T}_{\gamma}^{l, q}(\Omega)$, with $l \geq 2, q>\frac{3}{2}$ and assume that the weight index $\gamma$ satisfies (2.4). Moreover, let $(\mathbf{v}, 0) \in \mathfrak{D}_{\gamma}^{l, q} V(\Omega)$, with

$$
\left\|(\mathbf{v}, 0) ; \mathfrak{D}_{\gamma}^{l, q} V(\Omega)\right\| \leq \varepsilon,
$$

for some $\varepsilon>0$. Then, if $\varepsilon$ is chosen sufficiently small, problem (4.2) admits a unique solution $\widetilde{\mathbf{z}} \in V_{\gamma}^{l, q}(\Omega)^{3 \times 3}$ and the estimate

$$
\left\|\widetilde{\mathbf{z}} ; V_{\gamma}^{l, q}(\Omega)^{3 \times 3}\right\| \leq c\left\|\mathbf{g} ; \mathfrak{T}_{\gamma}^{l, q}(\Omega)\right\|
$$

holds. 
Proof. First, let us observe that since $\gamma-l<3-\frac{3}{q}$, it holds $r^{-1} \mathbf{V}(\theta) \in$ $V_{\gamma-1}^{l+1, q}(\Omega)^{3}$. Therefore, it follows that $\mathbf{v} \in V_{\gamma-1}^{l+1, q}(\Omega)^{3}$ and

$$
\left\|\mathbf{v} ; V_{\gamma-1}^{l+1, q}(\Omega)^{3}\right\| \leq c\left\|(\mathbf{v}, 0) ; \mathfrak{D}_{\gamma}^{l, q} V(\Omega)\right\| .
$$

Now, Lemma 3.1 (ii), provides the embedding $V_{\gamma-1}^{l+1, q}(\Omega) \hookrightarrow \Lambda_{\gamma-2}^{l-\frac{3}{q}-\alpha, \alpha}(\Omega)$, with $\alpha \in(0,1)$. Since $q>\frac{3}{2}$, one easily sees that there exists $\alpha \in(0,1)$ such that $\mathbf{v} \in C_{0}^{l-1, \alpha}(\Omega)^{3}$. Moreover, one obtains from Lemma 3.1 (i) the embeddings $V_{\gamma-1}^{1, q}(\Omega) \hookrightarrow V_{\gamma-l-2+\frac{3}{q}}^{0,3}(\Omega) \hookrightarrow L^{3}(\Omega)$, with the last inclusion following from the fact that $\gamma-l-2+\frac{3}{q}>0$. Hence, it holds

$$
\left\|D_{x}^{l} \mathbf{v} ; L^{3}(\Omega)^{3}\right\| \leq c\left\|D_{x}^{l} \mathbf{v} ; V_{\gamma-1}^{1, q}(\Omega)^{3}\right\| \leq c\left\|\mathbf{v} ; V_{\gamma-1}^{l+1, q}(\Omega)^{3}\right\|
$$

and one concludes that

$$
\left\|D_{x}^{l} \mathbf{v} ; L^{3}(\Omega)^{3}\right\|+\left\|\mathbf{v} ; C_{0}^{l-1, \alpha}(\Omega)^{3}\right\| \leq c\left\|(\mathbf{v}, 0) ; \mathfrak{D}_{\gamma}^{l, q} V(\Omega)\right\| .
$$

Next, let us write $\hat{\mathbf{g}}=\widetilde{\mathbf{g}}-\lambda_{1} \mathbf{v} \cdot \nabla\left(r^{-2} \mathbf{G}(\theta)\right)$ and observe that

$$
\mathbf{v} \cdot \nabla\left(r^{-2} \mathbf{G}(\theta)\right)=r^{-3}\left(-2 v_{r}(r, \theta)+v_{\theta}(r, \theta) \frac{\partial}{\partial \theta}\right) \mathbf{G}(\theta)
$$

where $\mathbf{v}(r, \theta)=\left(v_{r}(r, \theta), v_{\theta}(r, \theta)\right)$. On the other hand, since $\mathbf{v} \in V_{\gamma-1}^{l+1, q}(\Omega)^{3}$ it follows that $r^{-3} \mathbf{v} \in V_{\gamma+2}^{l+1, q}(\Omega)^{3}$. In particular, it holds $r^{-3} \mathbf{v} \in W^{l+1, q}(\Omega)^{3}$. Recalling that for $l \geq 2$ and $q>\frac{3}{2}$ the spaces $W^{l, q}(\Omega)^{3}$ form a Banach algebra, one obtains

$$
\begin{aligned}
\left\|\mathbf{v} \cdot \nabla\left(r^{-2} \mathbf{G}(\theta)\right) ; V_{\gamma}^{l, q}(\Omega)^{3 \times 3}\right\| & \leq c\left\|r^{-3} \mathbf{v} ; W^{l+1, q}(\Omega)^{3}\right\|\left\|\mathbf{G}(\theta) ; W^{l+1, q}(\Omega)^{3 \times 3}\right\| \\
& \leq c\left\|\mathbf{v} ; V_{\gamma-1}^{l+1, q}(\Omega)^{3}\right\|\left\|\mathbf{G}(\theta) ; W^{l+1, q}(\Omega)^{3 \times 3}\right\| .
\end{aligned}
$$

Hence, one concludes that $\hat{\mathbf{g}} \in V_{\gamma}^{l, q}(\Omega)^{3 \times 3}$ and

$$
\begin{aligned}
\left\|\hat{\mathbf{g}} ; V_{\gamma}^{l, q}(\Omega)^{3 \times 3}\right\| & \leq c\left(\left\|\widetilde{\mathbf{g}} ; V_{\gamma}^{l, q}(\Omega)^{3 \times 3}\right\|+\left\|\mathbf{v} ; V_{\gamma-1}^{l+1, q}(\Omega)^{3}\right\|\left\|\mathbf{G} ; W^{l+1, q}(\mathbb{S})^{3 \times 3}\right\|\right) \\
& \leq c\left\|\mathbf{g} ; \mathfrak{T}_{\gamma}^{l, q}(\Omega)\right\| .
\end{aligned}
$$

Now, since $q>\frac{3}{2}$ it follows from (2.4) that $\gamma>l$. Hence, it holds $\hat{\mathrm{g}} \in W^{l, q}(\Omega)^{3 \times 3}$. Therefore, in view of Lemma 4.1 one deduces that, for sufficiently small $\varepsilon>0$, there exists a unique solution $\widetilde{\mathbf{z}} \in W^{l, q}(\Omega)^{3 \times 3}$ to problem (4.2).

Next, let $R_{t}$ be a function such that

$$
R_{t}(r)= \begin{cases}r, & r<t \\ t, & r>t .\end{cases}
$$


Multiplying Equation (4.2) by $R^{q(\gamma-l)} \widetilde{\mathbf{z}}|\widetilde{\mathbf{z}}|^{q-2}$ and integrating over $\Omega$ (note that all integrals converge) yields

$$
\begin{aligned}
& \int_{\Omega} R_{t}^{q(\gamma-l)}|\widetilde{\mathbf{z}}|^{q} d x+\lambda_{1} \int_{\Omega} R_{t}^{q(\gamma-l)} \widetilde{\mathbf{z}}|\widetilde{\mathbf{z}}|^{q-2} \mathbf{v} \cdot \nabla \widetilde{\mathbf{z}} d x \\
& =\int_{\Omega} R_{t}^{\gamma-l} \hat{\mathrm{g}} R_{t}^{(q-1)(\gamma-l)} \widetilde{\mathbf{z}}|\widetilde{\mathbf{z}}|^{q-2} d x,
\end{aligned}
$$

where $|\widetilde{\mathbf{z}}|=\left(\sum_{i, j=1}^{3} z_{i j}^{2}\right)^{1 / 2}$. Integrating by parts and using Hölder inequality, it follows that

$$
\begin{aligned}
\int_{\Omega} R_{t}^{q(\gamma-l)}|\widetilde{\mathbf{z}}|^{q} d x \leq & c\left\|\mathbf{v} ; C_{0}^{l-1, \alpha}(\Omega)^{3}\right\| \int_{\Omega_{t}} R_{t}^{q(\gamma-l)-1}|\widetilde{\mathbf{z}}|^{q} d x \\
& +\left(\int_{\Omega} R_{t}^{q(\gamma-l)}|\hat{\mathbf{g}}|^{q} d x\right)^{1 / q}\left(\int_{\Omega} R_{t}^{q(\gamma-l)}|\widetilde{\mathbf{z}}|^{q} d x\right)^{(q-1) / q} .
\end{aligned}
$$

Hence, for $\left\|\mathbf{v} ; C_{0}^{l-1, \alpha}(\Omega)^{3}\right\|$ sufficiently small, one deduces that

$$
\left(\int_{\Omega} R_{t}^{q(\gamma-l)}|\widetilde{\mathbf{z}}|^{q} d x\right)^{1 / q} \leq c\left(\int_{\Omega} R_{t}^{q(\gamma-l)}|\hat{\mathbf{g}}|^{q} d x\right)^{1 / q},
$$

and letting $t \rightarrow \infty$, one gets

$$
\left\|\widetilde{\mathbf{z}} ; L_{\gamma}^{q}(\Omega)^{3 \times 3}\right\| \leq c\left\|\hat{\mathbf{g}} ; L_{\gamma}^{q}(\Omega)^{3 \times 3}\right\| .
$$

In the same manner, differentiating Equation (4.1) with respect to $x$, one obtains

$$
\begin{aligned}
& \left\|D_{x}^{k} \widetilde{\mathbf{z}} ; L_{\gamma}^{q}(\Omega)^{3 \times 3}\right\| \\
& \leq c\left(\left\|\widetilde{\mathbf{z}} ; V_{\gamma}^{k-1, q}(\Omega)^{3 \times 3}\right\|+\left\|D_{x}^{k} \hat{\mathbf{g}} ; L_{\gamma}^{q}(\Omega)^{3 \times 3}\right\|\right), \quad \forall k=1, \ldots, l-1,
\end{aligned}
$$

if $\left\|\mathbf{v} ; C_{0}^{l-1, \alpha}(\Omega)^{3}\right\|$ is chosen sufficiently small.

Finally, in order to estimate the $L_{\gamma}^{q}(\Omega)$-norm of $D_{x}^{l} \widetilde{\mathbf{z}}$, one first extends $\widetilde{\mathbf{z}}, \mathbf{v}$ and $\hat{\mathbf{g}}$ continuously to $\mathbb{R}^{3}$, then applies the operator $D_{x}^{l}$ to the equation in $\mathbb{R}^{3}$ and finally mollifies the resulting equation in order to integrate by parts in the term $\int_{\Omega} R^{q(\gamma-l)} \widetilde{\mathbf{z}}|\widetilde{\mathbf{z}}|^{q-2} \mathbf{v} \cdot \nabla D_{x}^{l} \widetilde{\mathbf{z}} d x$. The standard properties of the mollifier then yield the estimate

$$
\left\|D_{x}^{l} \widetilde{\mathbf{z}} ; L_{\gamma}^{q}(\Omega)^{3 \times 3}\right\| \leq c\left(\left\|\widetilde{\mathbf{z}} ; V_{\gamma}^{l-1, q}(\Omega)^{3 \times 3}\right\|+\left\|D_{x}^{l} \hat{\mathbf{g}} ; V_{\gamma}^{l, q}(\Omega)^{3 \times 3}\right\|\right),
$$

provided $\left\|\mathbf{v} ; C_{0}^{l-1, \alpha}(\Omega)^{3}\right\|+\left\|\mathbf{v} ; L^{3}(\Omega)^{3}\right\|$ is chosen small enough. From (4.4), (4.5) and (4.6), one concludes that

$$
\left\|\widetilde{\mathbf{z}} ; V_{\gamma}^{l, q}(\Omega)^{3 \times 3}\right\| \leq c\left\|\hat{\mathbf{g}} ; V_{\gamma}^{l, q}(\Omega)^{3 \times 3}\right\| .
$$




\section{Considering the equations of viscoelastic fluids.}

We are now ready to announce the main results. Since the structure of the decomposed problems (1.1) and (1.4) is very similar, we shall give the detailed proofs only for the Oldroyd-B fluid. For the second-grade fluid equations, we concentrate on the question of presenting the results in terms of the original variables $(\mathbf{v}, p)$ of the starting problem (1.3).

\subsection{Oldroyd-B fluid.}

Theorem 5.1. Let $\left(\mathbf{f}, 0, \mathbf{v}_{*}\right) \in \mathfrak{R}_{\gamma}^{l, q} V(\Omega)_{\perp}$, with $l \geq 2, \frac{3}{2}<q<\infty, \gamma-l \in$ $\left(2-\frac{3}{q}, 3-\frac{3}{q}\right)$ and

$$
\left\|\left(\mathbf{f}, 0, \mathbf{v}_{*}\right) ; \mathfrak{R}_{\gamma}^{l, q} V(\Omega)\right\| \leq \epsilon .
$$

There exists $\omega_{0} \in(0,1)$ such that for all $\omega \in\left(0, \omega_{0}\right]$ and for sufficiently small $\epsilon>0$, problem (1.1) admits a unique solution $((\mathbf{v}, p), \boldsymbol{\tau}) \in \mathfrak{D}_{\gamma}^{l, q} V(\Omega) \times$ $\mathfrak{T}_{\gamma}^{l, q}(\Omega)$ satisfying the estimate

$$
\left\|(\mathbf{v}, p) ; \mathfrak{D}_{\gamma}^{l, q} V(\Omega)\right\|+\left\|\boldsymbol{\tau} ; \mathfrak{T}_{\gamma}^{l, q}(\Omega)\right\| \leq c\left\|\left(\mathbf{f}, 0, \mathbf{v}_{*}\right) ; \mathfrak{R}_{\gamma}^{l, q} V(\Omega)\right\| .
$$

Proof. Take $((\boldsymbol{\varphi}, \xi), \boldsymbol{\psi}) \in \mathfrak{D}_{\gamma}^{l, q} V(\Omega) \times \mathfrak{T}_{\gamma}^{l, q}(\Omega)$, where $\boldsymbol{\varphi}$ is solenoidal in $\Omega$, and consider the Stokes problem

$$
\begin{cases}-\nu_{0}(1-\omega) \Delta \mathbf{v}+\nabla p=\nabla \cdot \boldsymbol{\psi}+\boldsymbol{\varphi} \cdot \nabla \boldsymbol{\varphi}+\mathbf{f} & \text { in } \Omega \\ \nabla \cdot \mathbf{v}=0 & \text { on } \partial \Omega \\ \mathbf{v}=\mathbf{v}_{*}, \quad \mathbf{v}_{*} \cdot \mathbf{n}=0 & \\ \lim _{|x| \rightarrow \infty} \mathbf{v}(x)=0 . & \end{cases}
$$

Observe that $(\nabla \cdot \boldsymbol{\psi}, 0,0) \in \mathfrak{R}_{\gamma}^{l, q} V(\Omega)$ and

$$
\left\|(\nabla \cdot \boldsymbol{\psi}, 0,0) ; \mathfrak{R}_{\gamma}^{l, q} V(\Omega)\right\| \leq\left\|\boldsymbol{\psi} ; \mathfrak{T}_{\gamma}^{l, q}(\Omega)\right\| .
$$

Therefore, since Lemmas 3.2 and 3.4, and Corollary 3.1 show that

$$
(\nabla \cdot \boldsymbol{\psi}+\boldsymbol{\varphi} \cdot \nabla \boldsymbol{\varphi}, 0,0) \in \mathfrak{R}_{\gamma}^{l, q} V(\Omega)_{\perp},
$$

one concludes from Theorem 2.3 that there exists a unique solution $(\mathbf{v}, p) \in$ $\mathfrak{D}_{\gamma}^{l, q} V(\Omega)$ to problem (5.2) satisfying the estimate

$$
\begin{aligned}
& \left\|(\mathbf{v}, p) ; \mathfrak{D}_{\gamma}^{l, q} V(\Omega)\right\| \\
& \leq \frac{c}{1-\omega}\left(\left\|\left(\mathbf{f}, 0, \mathbf{v}_{*}\right) ; \mathfrak{R}_{\gamma}^{l, q} V(\Omega)\right\|+\left\|\boldsymbol{\psi} ; \mathfrak{T}_{\gamma}^{l, q}(\Omega)\right\|+\left\|(\boldsymbol{\varphi}, \xi) ; \mathfrak{D}_{\gamma}^{l, q} V(\Omega)\right\|^{2}\right) .
\end{aligned}
$$


Next, consider the transport equation

$$
\boldsymbol{\tau}+\lambda_{1} \mathbf{v} \cdot \nabla \boldsymbol{\tau}=2 \nu_{0} \omega \mathbf{D}(\mathbf{v})+\mathbf{N}_{1}(\boldsymbol{\psi}, \nabla \boldsymbol{\varphi}) \text { in } \Omega
$$

where $(\mathbf{v}, 0) \in \mathfrak{D}_{\gamma}^{l, q} V(\Omega)$ is the solution of the Stokes system (5.2). Since $\mathbf{D}(\mathbf{v}) \in \mathfrak{T}_{\gamma}^{l, q}(\Omega)$, one sees by Corollary 3.2 and Lemma 4.2 that if the norm $\left\|(\mathbf{v}, 0) ; \mathfrak{D}_{\gamma}^{l, q} V(\Omega)\right\|$ is chosen sufficiently small then there exists a unique solution $\boldsymbol{\tau} \in \mathfrak{T}_{\gamma}^{l, q}(\Omega)$ to problem (5.4) such that

$$
\begin{aligned}
& \left\|\boldsymbol{\tau} ; \mathfrak{T}_{\gamma}^{l, q}(\Omega)\right\| \\
& \leq c\left(\omega\left\|(\mathbf{v}, 0) ; \mathfrak{D}_{\gamma}^{l, q} V(\Omega)\right\|+\left\|(\boldsymbol{\varphi}, 0) ; \mathfrak{D}_{\gamma}^{l, q} V(\Omega)\right\|\left\|\boldsymbol{\psi} ; \mathfrak{T}_{\gamma}^{l, q}(\Omega)\right\|\right) .
\end{aligned}
$$

Now, let us set

$$
\mathbb{B}_{\varepsilon}=\left\{((\boldsymbol{\varphi}, \xi), \boldsymbol{\psi}):\left\|(\boldsymbol{\varphi}, \xi) ; \mathfrak{D}_{\gamma}^{l, q} V(\Omega)\right\|+\left\|\boldsymbol{\psi} ; \mathfrak{T}_{\gamma}^{l, q}(\Omega)\right\| \leq \varepsilon\right\}
$$

and assume that $((\boldsymbol{\varphi}, \xi), \boldsymbol{\psi}) \in \mathbb{B}_{\varepsilon}$. Estimates (5.3) and (5.5) imply that for sufficiently small $\varepsilon$ one may define a mapping

$$
\mathcal{A}:((\boldsymbol{\varphi}, \xi), \boldsymbol{\psi}) \in \mathfrak{D}_{\gamma}^{l, q} V(\Omega) \times \mathfrak{T}_{\gamma}^{l, q}(\Omega) \longrightarrow((\mathbf{v}, p), \boldsymbol{\tau}) \in \mathfrak{D}_{\gamma}^{l, q} V(\Omega) \times \mathfrak{T}_{\gamma}^{l, q}(\Omega)
$$

through problems (5.2) and (5.4). Taking estimates (5.3) and (5.5) into account, one also sees that there exist $\omega_{0} \in(0,1)$ and $\varepsilon_{0}>0$ such that, for all $\omega \in\left(0, \omega_{0}\right]$ and $\varepsilon \in\left(0, \varepsilon_{0}\right]$, the mapping $\mathcal{A}$ maps the ball $\mathbb{B}_{\varepsilon}$ into itself.

Next, take

$$
\left(\left(\boldsymbol{\varphi}_{1}, \xi_{1}\right), \boldsymbol{\psi}_{1}\right),\left(\left(\boldsymbol{\varphi}_{2}, \xi_{2}\right), \boldsymbol{\psi}_{2}\right) \in \mathbb{B}_{\varepsilon}
$$

with $\varepsilon \in\left(0, \varepsilon_{0}\right]$ and let $\left(\left(\mathbf{v}_{1}, p_{1}\right), \boldsymbol{\tau}_{1}\right)$ and $\left(\left(\mathbf{v}_{2}, p_{2}\right), \boldsymbol{\tau}_{2}\right)$ be the corresponding solutions to problems (5.2) and (5.4). Setting

$$
\begin{gathered}
\boldsymbol{\varphi}=\boldsymbol{\varphi}_{1}-\boldsymbol{\varphi}_{2}, \quad \xi=\xi_{1}-\xi_{2}, \quad \boldsymbol{\psi}=\boldsymbol{\psi}_{1}-\boldsymbol{\psi}_{2}, \\
\mathbf{v}=\mathbf{v}_{1}-\mathbf{v}_{2}, \quad p=p_{1}-p_{2},
\end{gathered}
$$

one easily sees that $(\mathbf{v}, p)$ satisfies a Stokes problem for which Lemma 2.3 provides the estimate

$$
\begin{aligned}
& \left\|(\mathbf{v}, p) ; \mathfrak{D}_{\gamma-1}^{l-1, q} V(\Omega)\right\| \\
& \leq \frac{c}{1-\omega}\left(\left\|\boldsymbol{\psi} ; \mathfrak{T}_{\gamma-1}^{l-1, q}(\Omega)\right\|+\varepsilon\left\|(\boldsymbol{\varphi}, \xi) ; \mathfrak{D}_{\gamma-1}^{l-1, q} V(\Omega)\right\|\right) .
\end{aligned}
$$

In particular, note that since $l \geq 2$, the term

$$
\boldsymbol{\varphi}_{1} \cdot \nabla \boldsymbol{\varphi}_{1}-\boldsymbol{\varphi}_{2} \cdot \nabla \boldsymbol{\varphi}_{2}=\boldsymbol{\varphi}_{1} \cdot \nabla \boldsymbol{\varphi}+\boldsymbol{\varphi} \cdot \nabla \boldsymbol{\varphi}_{2}
$$

can be directly estimated in $\Re_{\gamma-1}^{l-1, q} V(\Omega)$ by Lemma 3.2 where $l, \gamma$ are changed to $l-1, \gamma-1$ (note that condition (2.4) still remains valid).

Now, set $\boldsymbol{\tau}=\boldsymbol{\tau}_{1}-\boldsymbol{\tau}_{2}$ and observe that $\boldsymbol{\tau}$ satisfies the following transport equation

$$
\boldsymbol{\tau}+\lambda_{1} \mathbf{v}_{1} \cdot \nabla \boldsymbol{\tau}=2 \nu_{0} \omega \mathbf{D}(\mathbf{v})-\lambda_{1} \mathbf{v} \cdot \nabla \boldsymbol{\tau}_{2}+\mathbf{N}_{1}\left(\boldsymbol{\psi}_{1}, \nabla \boldsymbol{\varphi}_{1}\right)-\mathbf{N}_{1}\left(\boldsymbol{\psi}_{2}, \nabla \boldsymbol{\varphi}_{2}\right) .
$$


As in Lemma 2.3, for $(\mathbf{v}, 0) \in \mathfrak{D}_{\gamma-1}^{l-1, q} V(\Omega)$ and $\boldsymbol{\tau}_{2} \in \mathfrak{T}_{\gamma}^{l, q}(\Omega)$, we conclude that

$$
\mathbf{v} \in V_{\beta-1}^{l, q}(\Omega)^{3}, \quad \boldsymbol{\tau}_{2} \in V_{\beta}^{l, q}(\Omega)^{3 \times 3},
$$

for any $\beta-l \in\left(1-\frac{3}{q}, 2-\frac{3}{q}\right)$. Moreover, embeddings of Lemma 3.1 (ii) yield

$$
\mathbf{v} \in \Lambda_{\beta-1}^{l-\frac{3}{q}-\alpha, \alpha}(\Omega)^{3}, \quad \boldsymbol{\tau}_{2} \in \Lambda_{\beta}^{l-\frac{3}{q}-\alpha, \alpha}(\Omega)^{3 \times 3},
$$

where $\alpha \in(0,1)$ is chosen in such a way that $l-\frac{3}{q}-\alpha$ becomes a nonnegative integer. To prove that $\mathbf{v} \cdot \nabla \boldsymbol{\tau}_{2} \in V_{\gamma-1}^{l-1, q}(\Omega)^{3 \times 3}$, we need to verify that

$$
r^{\gamma-l+k} D_{x}^{k}\left(\mathbf{v} \cdot \nabla \boldsymbol{\tau}_{2}\right) \in L^{q}(\Omega),
$$

for $k=0, \ldots, l-1$, or equivalently

$$
{ }^{\gamma-l+k} D_{x}^{s} \mathbf{v} \cdot D_{x}^{k-s} \nabla \boldsymbol{\tau}_{2} \in L^{q}(\Omega),
$$

with $s=0, \ldots, k$.

First, let $l \geq 3$. Since $q>\frac{3}{2}$, from (5.7) it follows that

$$
\mathbf{v} \in \Lambda_{\beta-3+\frac{3}{q}+\alpha}^{l-2, \alpha}(\Omega)^{3}, \quad \nabla \boldsymbol{\tau}_{2} \in \Lambda_{\beta-l+\frac{3}{q}+\alpha+1}^{0, \alpha}(\Omega)^{3 \times 3 \times 3} .
$$

Thus, if $s \leq l-2$, then

$$
r^{\beta-3+\frac{3}{q}-(l-2)+s}\left|D_{x}^{s} \mathbf{v}(x)\right| \text { is bounded in } \Omega .
$$

Therefore, it suffices to prove that

$$
r^{\gamma-l+k} r^{-\left(\beta-1+\frac{3}{q}-l+s\right)} D_{x}^{k-s} \nabla \boldsymbol{\tau}_{2} \in L^{q}(\Omega) .
$$

Now, since $\boldsymbol{\tau}_{2} \in V_{\beta}^{l, q}(\Omega)^{3 \times 3}$ it follows in particular that

$$
r^{\beta-l+k-s+1} D_{x}^{k-s} \nabla \boldsymbol{\tau}_{2} \in L^{q}(\Omega), \quad k=0, \ldots, l-1, \quad s=0, \ldots, k .
$$

On the other hand, fixing $\beta=\gamma-1$ and recalling that $\gamma-l \geq 2-\frac{3}{q}$, one easily sees that

$$
\gamma-l+k-\left(\beta-1+\frac{3}{q}-l+s\right) \leq \beta-l+k-s+1,
$$

and (5.9) follows. If $s=k=l-1$, one gets from (5.7) that

$$
r^{\beta-l+\frac{3}{q}+1}\left|\nabla \boldsymbol{\tau}_{2}(x)\right| \text { is bounded in } \Omega \text {. }
$$

Hence, it remains to be shown that

$$
r^{\gamma-l+(l-1)} r^{-\left(\beta-l+\frac{3}{q}+1\right)} D_{x}^{l-1} \mathbf{v} \in L^{q}(\Omega) .
$$

Since $r^{\beta-1-l+(l-1)} D_{x}^{l-1} \mathbf{v} \in L^{q}(\Omega)$ and since for $\beta=\gamma-1$ it holds $\beta-2 \geq$ $\gamma-1-\left(\beta-l+\frac{3}{q}+1\right)$, inclusion (5.10) is valid. Hence, (5.8) has been verified for $l \geq 3$. 
For $l=2$, the result is proven in a similar way, except for the inclusion

$$
r^{\gamma-1} D_{x} \mathbf{v} \cdot \nabla \boldsymbol{\tau}_{2} \in L^{q}(\Omega)
$$

for which one uses the embeddings of Lemma 3.1 (i), namely

$$
\nabla \mathbf{v} \in V_{\beta-1}^{1, q}(\Omega) \hookrightarrow V_{\beta-2+\frac{3}{2 q}}^{0,2 q}(\Omega), \quad \nabla \boldsymbol{\tau}_{2} \in V_{\beta}^{1, q}(\Omega) \hookrightarrow V_{\beta-1+\frac{3}{2 q}}^{0,2 q}(\Omega) .
$$

Observing that

$$
r^{\gamma-1} D_{x} \mathbf{v} \cdot \boldsymbol{\tau}_{2}=r^{\gamma-2 \beta+2-\frac{3}{q}} r^{\beta-2-\frac{3}{2 q}} D_{x} \mathbf{v} \cdot r^{\beta-1-\frac{3}{2 q}} \nabla \boldsymbol{\tau}_{2}
$$

and that for $\beta=\gamma-1$ it holds $\gamma-2 \beta+2-\frac{3}{q} \leq 0$, one easily sees using Hölder's inequality that (5.11) is valid. Hence, one concludes that $\mathbf{v} \cdot \nabla \boldsymbol{\tau}_{2} \in$ $V_{\gamma-1}^{l-1, q}(\Omega)^{3 \times 3}$ and

$$
\left\|\mathbf{v} \cdot \nabla \boldsymbol{\tau}_{2} ; V_{\gamma-1}^{l-1, q}(\Omega)^{3 \times 3}\right\| \leq c\left\|(\mathbf{v}, 0) ; \mathfrak{D}_{\gamma-1}^{l-1, q} V(\Omega)\right\|\left\|\boldsymbol{\tau}_{2} ; \mathfrak{T}_{\gamma}^{l, q}(\Omega)\right\| .
$$

In a similar manner, see also Lemma 3.5 and Corollary 3.2 which yield the result for $l \geq 3$, one shows that

$$
\begin{aligned}
& \left\|\mathbf{N}_{1}\left(\boldsymbol{\psi}_{1}, \nabla \boldsymbol{\varphi}_{1}\right)-\mathbf{N}_{1}\left(\boldsymbol{\psi}_{2}, \nabla \boldsymbol{\varphi}_{2}\right) ; V_{\gamma-1}^{l-1, q}(\Omega)\right\| \\
& \leq c\left(\left\|\boldsymbol{\psi}_{1} ; \mathfrak{T}_{\gamma}^{l, q}(\Omega)\right\|\left\|(\boldsymbol{\varphi}, \xi) ; \mathfrak{D}_{\gamma-1}^{l-1, q} V(\Omega)\right\|\right. \\
& \left.\quad+\left\|\left(\boldsymbol{\varphi}_{2}, \xi_{2}\right) ; \mathfrak{D}_{\gamma}^{l, q} V(\Omega)\right\|\left\|\boldsymbol{\psi} ; \mathfrak{T}_{\gamma-1}^{l-1, q}(\Omega)\right\|\right) .
\end{aligned}
$$

Hence, in view of Lemma 4.2 one finally concludes that

$$
\begin{aligned}
\left\|\boldsymbol{\tau} ; \mathfrak{T}_{\gamma-1}^{l-1, q}(\Omega)\right\| \leq & c\left((\omega+\varepsilon)\left\|(\mathbf{v}, 0) ; \mathfrak{D}_{\gamma-1}^{l-1, q} V(\Omega)\right\|\right. \\
& \left.+\varepsilon\left(\left\|(\boldsymbol{\varphi}, \xi) ; \mathfrak{D}_{\gamma-1}^{l-1, q} V(\Omega)\right\|+\left\|\boldsymbol{\psi} ; \mathfrak{T}_{\gamma-1}^{l-1, q}(\Omega)\right\|\right)\right),
\end{aligned}
$$

where we have taken into account that $\left(\left(\boldsymbol{\varphi}_{1}, \xi_{1}\right), \boldsymbol{\psi}_{1}\right),\left(\left(\boldsymbol{\varphi}_{2}, \xi_{2}\right), \boldsymbol{\psi}_{2}\right) \in \mathbb{B}_{\varepsilon}$ and that $\mathcal{A}\left(\mathbb{B}_{\varepsilon}\right) \subset \mathbb{B}_{\varepsilon}$.

Estimates (5.6) and (5.12) together show that, for small enough $\omega>0$ and $\varepsilon>0$, the mapping $\mathcal{A}$ is a contraction in the topology of $\mathfrak{D}_{\gamma-1}^{l-1, q} V(\Omega) \times$ $\mathfrak{T}_{\gamma-1}^{l-1, q}(\Omega)$. Let us point out once more that the space $\mathfrak{D}_{\gamma-1}^{l-1, q} V(\Omega) \times \mathfrak{T}_{\gamma-1}^{l-1, q}(\Omega)$ naturally includes the original product space $\mathfrak{D}_{\gamma}^{l, q} V(\Omega) \times \mathfrak{T}_{\gamma}^{l, q}(\Omega)$. Consequently, $\mathcal{A}$ has a unique fixed point $((\mathbf{v}, p), \boldsymbol{\tau}) \in \mathfrak{D}_{\gamma}^{l, q} V(\Omega) \times \mathfrak{T}_{\gamma}^{l, q}(\Omega)$, i.e., problem (1.1) has a unique solution $((\mathbf{v}, p), \boldsymbol{\tau}) \in \mathfrak{D}_{\gamma}^{l, q} V(\Omega) \times \mathfrak{T}_{\gamma}^{l, q}(\Omega)$. Estimate (5.1) follows from (5.3) and (5.5) written for the fixed point.

5.2. Second-grade fluid. Using Theorems 2.3 and 4.2 , Lemma 3.3 and Corollary 3.3 and arguing in a similar way as in the proof of Theorem 5.1, one obtains the following result. 
Theorem 5.2. Let $\mathcal{F} \in \mathfrak{T}_{\gamma}^{l, q}(\Omega)$ and $\mathbf{v}_{*} \in W^{l+1-1 / q, q}(\partial \Omega)$, with $l \geq 2, \frac{3}{2}<$ $q<\infty$ and $\gamma-l \in\left(2-\frac{3}{q}, 3-\frac{3}{q}\right)$. There exists a positive constant $\epsilon>0$ such that if

$$
\left\|\mathcal{F} ; \mathfrak{T}_{\gamma}^{l, q}(\Omega)\right\|+\left\|\mathbf{v}_{*} ; W^{l+1-1 / q, q}(\partial \Omega)\right\| \leq \epsilon,
$$

then problem (1.4) admits a unique solution $((\mathbf{v}, \pi), \mathbf{z}) \in \mathfrak{D}_{\gamma}^{l, q} V(\Omega) \times \mathfrak{T}_{\gamma}^{l, q}(\Omega)$ satisfying the estimate

$$
\begin{aligned}
& \left\|(\mathbf{v}, \pi) ; \mathfrak{D}_{\gamma}^{l, q} V(\Omega)\right\|+\left\|\mathbf{z} ; \mathfrak{T}_{\gamma}^{l, q}(\Omega)\right\| \\
& \leq c\left(\left\|\mathcal{F} ; \mathfrak{T}_{\gamma}^{l, q}(\Omega)\right\|+\left\|\mathbf{v}_{*} ; W^{l+1-1 / q, q}(\partial \Omega)\right\|\right) .
\end{aligned}
$$

In order to come back to the original variables, we need the following two auxiliary results.

Lemma 5.1. Let $(\mathbf{v}, \pi) \in \mathfrak{D}_{\gamma}^{l, q} V(\Omega)$, with $l \geq 2, \frac{3}{2}<q<\infty$ and $\gamma-l \in(2-$ $\left.\frac{3}{q}, 3-\frac{3}{q}\right)$. The function $p$ defined by (1.6) has the asymptotic representation

$$
p(x)=r^{-2} P(\theta)+\widetilde{p}(x),
$$

where $P \in W^{l+1, q}\left(\mathbb{S}^{2}\right)$ and $\widetilde{p} \in V_{\gamma-1}^{l-1, q}(\Omega)$. Moreover, it holds

$$
\begin{aligned}
& \left\|P ; W^{l+1, q}\left(\mathbb{S}^{2}\right)\right\|+\left\|\widetilde{p} ; V_{\gamma-1}^{l-1, q}(\Omega)\right\| \\
& \leq c\left(\left\|(\mathbf{v}, \pi) ; \mathfrak{D}_{\gamma}^{l, q} V(\Omega)\right\|+\left\|(\mathbf{v}, \pi) ; \mathfrak{D}_{\gamma}^{l, q} V(\Omega)\right\|^{2}\right) .
\end{aligned}
$$

Proof. Since $(\mathbf{v}, \pi) \in \mathfrak{D}_{\gamma}^{l, q} V(\Omega)$, one can write

$$
\mathbf{v}(x)=r^{-1} \mathbf{V}(\theta)+\widetilde{\mathbf{v}}(x), \quad \pi(x)=r^{-2} \Pi(\theta)+\widetilde{\pi}(x),
$$

with $\mathbf{V} \in W^{l+2, q}\left(\mathbb{S}^{2}\right)^{3}, \widetilde{\mathbf{v}} \in V_{\gamma}^{l+1, q}(\Omega)^{3}, \Pi \in W^{l+1, q}\left(\mathbb{S}^{2}\right)$ and $\tilde{\pi} \in V_{\gamma}^{l, q}(\Omega)$. On the other hand, since $\gamma-l>2-\frac{3}{q}$, one easily shows that $r^{-1} \mathbf{V}(\theta) \in$ $V_{\gamma-1}^{l+1, q}(\Omega)^{3}, \nabla_{x}\left(r^{-2} \Pi(\theta)\right) \in V_{\gamma}^{l, q}(\Omega)^{3}$ and $\nabla \widetilde{\pi} \in V_{\gamma}^{l-1, q}(\Omega)^{3}$. Using the embeddings of Lemma 3.1, one concludes that $\mathbf{v} \cdot \nabla \pi \in V_{\gamma}^{l-1, q}(\Omega)$, provided $l \geq 2, q>\frac{3}{2}$ and $\gamma-l>2-\frac{3}{q}$.

Lemma 5.2. Let $(\mathbf{f}, 0,0) \in \mathfrak{R}_{\gamma}^{l, q} V(\Omega)_{\perp}$, with $l \geq 2, \frac{3}{2}<q<\infty$ and $\gamma-l \in$ $\left(2-\frac{3}{q}, 3-\frac{3}{q}\right)$. There exists a tensor function $\mathcal{F} \in \mathfrak{T}_{\gamma}^{l, q}(\Omega)$ such that $\nabla \cdot \mathcal{F}=\mathbf{f}$. Moreover, it holds

$$
\left\|\mathcal{F} ; \mathfrak{T}_{\gamma}^{l, q}(\Omega)\right\| \leq c\left\|(\mathbf{f}, 0,0) ; \mathfrak{R}_{\gamma}^{l, q} V(\Omega)\right\| .
$$


Proof. Let us consider the following auxiliary problem for the Laplace operator in a three-dimensional exterior domain

$$
\begin{cases}-\Delta \mathbf{w}=\mathbf{f} & \text { in } \Omega \\ \mathbf{w}=\mathbf{h} & \text { on } \partial \Omega,\end{cases}
$$

with some given vector functions $\mathbf{f}$ and $\mathbf{h}$. In other words, we consider the Dirichlet problem for the scalar Poisson equation for each of the components $w_{1}, w_{2}$ and $w_{3}$ of $\mathbf{w}$. Let us associate to problem (5.14) a mapping

$$
\mathcal{L}_{\beta}^{l, q}: V_{\beta}^{l+1, q}(\Omega)^{3} \longrightarrow V_{\beta}^{l-1, q}(\Omega)^{3} \times W^{l+1-1 / q, q}(\partial \Omega)^{3}
$$

defined by $\mathbf{w} \mapsto(\mathbf{f}, \mathbf{h})=\mathcal{L}_{\beta}^{l, q} \mathbf{w}$. In a similar way as for the Stokes operator (see Remark 2.1), one can show that any nontrivial power solution $r^{\lambda} \mathbf{W}(\theta)$ of the Laplace equation

$$
-\Delta \mathbf{w}=0, \quad \text { in } \mathbb{R}^{3} \backslash\{0\}
$$

has an integer exponent $\lambda$, while for $\lambda=0$ and $\lambda=-1$, $\mathbf{W}$ becomes a constant vector. Now, using standard techniques described, e.g., in [19], we conclude that the operator $\mathcal{L}_{\beta}^{l, q}$ is an isomorphism if and only if $\beta-l \in$ $\left(1-\frac{3}{q}, 2-\frac{3}{q}\right.$ ) (cf. Theorem 2.1 and Remark 2.2). Next, as in Section 2.3, we introduce the asymptotic forms

$$
\begin{aligned}
& \mathbf{f}(x)=r^{-3} \mathbf{F}(\theta)+\widetilde{\mathbf{f}}(x), \\
& \mathbf{F} \in W^{l, q}\left(\mathbb{S}^{2}\right)^{3}, \\
& \widetilde{\mathbf{f}} \in V_{\gamma}^{l-1, q}(\Omega)^{3}, \\
& \mathbf{w}(x)=r^{-1} \mathbf{W}(\theta)+\widetilde{\mathbf{w}}(x), \\
& \mathbf{W} \in W^{l+2, q}\left(\mathbb{S}^{2}\right)^{3}, \\
& \widetilde{\mathbf{w}} \in V_{\gamma}^{l+1, q}(\Omega)^{3}
\end{aligned}
$$

or, equivalently, suggest that $(\mathbf{f}, 0, \mathbf{h}) \in \mathfrak{R}_{\gamma}^{l, q} V(\Omega)$ and $(\mathbf{w}, 0) \in \mathfrak{D}_{\gamma}^{l, q} V(\Omega)$. Arguing as in the proof of Theorem 2.3 and recalling that any power solution $r^{0} \mathbf{W}(\theta)$ of Equation (5.15) is constant, we see that provided condition $\gamma-l \in$ $\left(2-\frac{3}{q}, 3-\frac{3}{q}\right)$ is satisfied, the mapping

$$
\mathfrak{D}_{\gamma}^{l, q} V(\Omega) \ni(\mathbf{w}, 0) \longmapsto\left(-\Delta \mathbf{w}, \mathbf{w}_{\mid \partial \Omega}\right) \in \mathfrak{R}_{\gamma}^{l, q} V(\Omega)_{\perp}
$$

is an isomorphism. Here, as before, $\mathfrak{R}_{\gamma}^{l, q} V(\Omega)_{\perp}$ denotes the subspace of functions in $\mathfrak{R}_{\gamma}^{l, q} V(\Omega)$ satisfying the compatibility condition (2.9). Moreover, the estimate

$$
\left\|(\mathbf{w}, 0) ; \mathfrak{D}_{\gamma}^{l, q} V(\Omega)\right\| \leq c\left\|(\mathbf{f}, 0, \mathbf{h}) ; \mathfrak{R}_{\gamma}^{l, q} V(\Omega)\right\|
$$

is valid. Now, taking $\mathbf{h}=0$ and $\mathcal{F}=-\nabla \mathbf{w}$ in (5.14) and observing that $\nabla \mathbf{w} \in \mathfrak{T}_{\gamma}^{l, q}(\Omega)$, with

$$
\left\|\nabla \mathbf{w} ; \mathfrak{T}_{\gamma}^{l, q}(\Omega)\right\| \leq c\left\|(\mathbf{w}, 0) ; \mathfrak{D}_{\gamma}^{l, q} V(\Omega)\right\|,
$$

leads to the statement of the Lemma. 
Remark 5.1. Clearly, the representation of $\mathcal{F}$ in the previous Lemma is not unique; e.g., one can change the Dirichlet condition to the Neumann one. However, one can show by direct estimations that the solution $(\mathbf{v}, p)$ to the original problem (1.3) is unique, see [23].

Now, let us formulate the main theorem.

Theorem 5.3. Let $\left(\mathbf{f}, 0, \mathbf{v}_{*}\right) \in \mathfrak{R}_{\gamma}^{l, q} V(\Omega)_{\perp}$, with $l \geq 2, \frac{3}{2}<q<\infty$ and $\gamma-l \in\left(2-\frac{3}{q}, 3-\frac{3}{q}\right)$. There exists a positive constant $\epsilon>0$ such that if

$$
\left\|\left(\mathbf{f}, 0, \mathbf{v}_{*}\right) ; \mathfrak{R}_{\gamma}^{l, q} V(\Omega)\right\| \leq \epsilon,
$$

then problem (1.3) has a unique solution $(\mathbf{v}, p)$ admitting the asymptotic representation

$$
\mathbf{v}(x)=r^{-1} \mathbf{V}(\theta)+\widetilde{\mathbf{v}}(x), \quad p(x)=r^{-2} P(\theta)+\widetilde{p}(x),
$$

with $\mathbf{V} \in W^{l+2, q}\left(\mathbb{S}^{2}\right)^{3}, \widetilde{\mathbf{v}} \in V_{\gamma}^{l+1, q}(\Omega)^{3}, P \in W^{l+1, q}\left(\mathbb{S}^{2}\right)$ and $\tilde{p} \in V_{\gamma-1}^{l-1, q}(\Omega)$. Moreover, this solution satisfies the following estimate

$$
\begin{aligned}
& \left\|\mathbf{V} ; W^{l+2, q}\left(\mathbb{S}^{2}\right)^{3}\right\|+\left\|\widetilde{\mathbf{v}} ; V_{\gamma}^{l+1, q}(\Omega)^{3}\right\|+\left\|P ; W^{l+1, q}\left(\mathbb{S}^{2}\right)\right\|+\left\|\widetilde{p} ; V_{\gamma-1}^{l-1, q}(\Omega)\right\| \\
& \leq c\left\|\left(\mathbf{f}, 0, \mathbf{v}_{*}\right) ; \mathfrak{R}_{\gamma}^{l, q} V(\Omega)\right\| .
\end{aligned}
$$

Remark 5.2. Typically, the original pressure function $p$ in the equations of the second-grade fluid is always less regular than the pressure in the NavierStokes equations with the same right-hand side. This results from definition (1.6) of $p$ in terms of the solution of the decomposed problem (1.4). Notice, however, that in the solution obtained in Theorem 5.3 we "lose" regularity only in the remainder part $\widetilde{p}$ and not in the main asymptotic term $P$.

Acknowledgements. The financial support from CMA/IST (Lisbon), from CIM/Fundação Calouste Gulbenkian - Thematic Term on Theoretical and Computational Fluid Dynamics (Coimbra) and European Union FEDER/ PRAXIS (Project Nr. 2/2.1/MAT/380/94) is greatly acknowledged.

\section{References}

[1] W. Borchers and K. Pileckas, Existence, uniqueness and asymptotics of steady jets, Arch. Rational Mech. Analysis, 120 (1992), 1-49, MR 93j:35129, Zbl 0772.76021.

[2] D. Cioranescu and E.H. Ouazar, Existence and uniqueness for fluids of second grade, Collège de France Seminars, Pitman Research Notes in Mathematics, 109, Pitman, Boston, (1984), 178-197, MR 86b:76011, Zbl 0577.76012.

[3] V. Coscia and G.P. Galdi, Existence, uniqueness and stability of regular steady motions of a second-grade fluid, Int. J. Non-Linear Mech., 29 (1994), 493-506, MR 95j:76006, Zbl 0815.76006.

[4] J.E. Dunn and R.L. Fosdick, Thermodynamics, stability and boundedness of fluids of complexity 2 and fluids of second grade, Arch. Rational Mech. Anal., 56 (1974), 191-252, MR 50 \#3738, Zbl 0324.76001. 
[5] R. Finn, Estimates at infinity for stationary solutions of the Navier-Stokes equations, Bull. Math. Soc. Sci. Math. Phys. R. P. Roumaine (N.S.), 3 (1959), 387-418, MR 29 \#3770, Zbl 0106.39402.

[6] _ On the exterior stationary problem for the Navier-Stokes equations, and associated perturbation problems, Arch. Rational Mech. Anal., 19 (1965), 363-406, MR 32 \#298, Zbl 0149.44606.

[7] G.P. Galdi and K.R. Rajagopal, Slow motion of a body in a fluid of second grade, Int. J. Engng. Sci., 35 (1997), 33-54, MR 98i:76005, Zbl 0908.76007.

[8] G.P. Galdi, A. Sequeira and J.H. Videman, Steady motions of a second-grade fluid in an exterior domain, Adv. Math. Sci. Appl., 7 (1997), 977-995, MR 98j:35151, Zbl 0894.76002.

[9] C. Guillopé and J.-C. Saut, Existence and stability of steady flows of weakly viscoelastic fluids, Proc. Roy. Soc. Edinburgh, 119 (1991), 137-158, MR 92i:35100, Zbl 0850.76041.

[10] D.D. Joseph, Fluid Dynamics of Viscoelastic Liquids, Springer Verlag, New York, 1990, MR 91d:76003, Zbl 0698.76002.

[11] V.A. Kondratjev, Boundary value problems for elliptic equations in domains with conical or corner points, Trudy Moskov. Mat. Obshch., 16 (1967), 209-292; English Transl.: Trans. Moscow Math. Soc., 16 (1967).

[12] L.D. Landau and E.M. Lifschitz, Méchanique des Fluides, Mir, 1971, Zbl 0216.25801.

[13] V.G. Maz'ya and B.A. Plamenevskii, On the coefficients in the asymptotics of solutions of elliptic boundary value problems in domains with conical points, Math. Nachr., 76 (1977), 29-60; English Transl., Amer. Math. Soc. Transl., 123(2) (1984), 57-88, Zbl 0554.35036.

[14] Estimates in $L_{p}$ and Hölder classes and the Miranda-Agmon maximum principle for solutions of elliptic boundary value problems in domains with singular points on the boundary, Math. Nachr., 81 (1978), 25-82; English Transl., Amer. Math. Soc. Transl., 123(2) (1984), 1-56, MR 58 \#11886, Zbl 0554.35035.

[15] S.A. Nazarov, On the two-dimensional aperture problem for Navier-Stokes equations, C.R. Acad. Sci. Paris, Ser.1, 323 (1996), 699-703, MR 97k:35199, Zbl 0860.35096.

[16] - The Navier-Stokes problem in a two-dimensional domain with angular outlets to infinity, Zapiski Nauchn. Seminar POMI, 257 (1999), 207-227 (Russian), MR 2001d:35159.

[17] S.A. Nazarov and K. Pileckas, Asymptotics of solutions to the Navier-Stokes system in the exterior of a bounded body, Dokl. Ross. Akad. Nauk., 367 (1999), 461-463; English transl., Russ. Acad. Sci. Dokl. Math., 60 (1999), 133-135.

[18] _ On steady Stokes and Navier-Stokes problems with zero velocity at infinity in a three-dimensional exterior domain, J. Math. Kyoto Univ., 40(3) (2000), 475-492, MR 2001j:35226.

[19] S.A. Nazarov and B.A. Plamenevskii, Elliptic boundary value problems in domains with piecewise smooth boundaries, Walter de Gruyter and Co, Berlin, 1994, MR 95h:35001, Zbl 0806.35001.

[20] S.A. Nazarov, M. Specovius-Neugebauer and G. Thäter, Quiet flows for Stokes and Navier-Stokes problems in domains with cylindrical outlets to infinity, Kyushu J. Math., 53 (1999), 369-394, MR 2000i:35163, Zbl 0939.35140. 
[21] A. Novotný, On the steady transport equation, Málek, J., Nečas, J. and M. Rokyta (eds.), Advanced Topics in Theoretical Fluid Mechanics, Pitman Research Notes in Mathematics, 392, Addison Wesley Longman, (1998), 118-146, MR 2000e:35182, Zbl 0941.35079.

[22] A. Novotný, A. Sequeira and J.H. Videman, Existence of three-dimensional flows of second-grade fluids past an obstacle, Non. Analy., Theory, Methods \& Appli., 30 (1997), 3051-3058, MR 99a:76006, Zbl 0893.35106.

[23] _ Steady motions of viscoelastic fluids in 3-D exterior domains - existence, uniqueness and asymptotic behaviour, Arch. Rational Mech. Analysis, 149 (1999), 49-67, MR 2000g:76006, Zbl 0937.35148.

[24] J.G. Oldroyd, On the formulation of rheological equations of state, Proc. Roy. Soc. London, Ser. A, 200 (1950), 523-541, MR 11,703a.

[25] K. Pileckas, A. Sequeira and J.H. Videman, Steady flows of viscoelastic fluids in domains with outlets to infinity, J. Math. Fluid Mech., 2(3) (2000), 185-218, MR 2001g:76004.

[26] M. Pokorny, Asymptotic behaviour of solutions to certain partial differential equations describing the flow of fluids in unbounded domains, $\mathrm{PhD}$ Thesis, University of Toulon and Charles University of Prague, 1999.

[27] K.R. Rajagopal, Mechanics of non-Newtonian fluids, G.P. Galdi and J. Nečas (eds.), Recent Developments in Theoretical Fluid Mechanics, Pitman Research Notes in Mathematics, 291, Longman Scientific and Technical, (1993), 129-162, MR 95c:76005, Zbl 0818.76003.

[28] M. Renardy, Existence of slow steady flows of viscoelastic fluids with differential constitutive equations, ZAMM, 65 (1985), 449-451, MR 87c:76018, Zbl 0577.76014.

[29] W.R. Schowalter, Mechanics of Non-Newtonian Fluids, Pergamon Press, New York, 1978.

[30] M. Specovius-Neugebauer, Approximation of Stokes Problems in Unbounded Domains, Habilitation Thesis, Universität Paderborn, 1997.

[31] C. Truesdell and W. Noll, The Nonlinear Field Theories of Mechanics, $2^{\text {nd }}$ edition, Springer, Berlin, 1992, MR 94c:73002, Zbl 0315.73008.

[32] J.H. Videman, Mathematical Analysis of Viscoelastic Non-Newtonian Fluids, PhD Thesis, Instituto Superior Técnico, Lisbon, 1997.

Received May 4, 2000.

Department of Mathematics and Mechanics

St. Petersburg State University

BiblioteChNAYA PL., 2

198904 St. PetersburG

Russia

E-mail address: serna@snark.ipme.ru

Instituto Superior TÉcnico

Departamento de Matemática

Av. Rovisco Pais, 1

1049-001 LisBoA

Portugal 
E-mail address: adelia.sequeira@math.ist.utl.pt

Instituto Superior TÉCNICO

Departamento de Matemática

Av. Rovisco Pais, 1

1049-001 LisBoA

PorTugal

E-mail address: videman@math.ist.utl.pt 